\title{
Symmetries and conserved quantities of constrained mechanical systems
}

\author{
F. X. Mei · H. B. Wu - Y. F. Zhang
}

Received: 20 October 2013 / Revised: 15 November 2013 / Accepted: 18 November 2013 / Published online: 10 December 2013

(C) Springer-Verlag Berlin Heidelberg 2013

\begin{abstract}
This is a concise review of the symmetries and conserved quantities of the constrained mechanical systems. It deals with three kinds of symmetries: the Noether symmetry, the Lie symmetry and the form invariance, and three kinds of conserved quantities: the Noether conserved quantity, the Hojman type conserved quantity and the new conserved quantity.
\end{abstract}

Keywords Symmetry · Conserved quantity ·

Constrained mechanical system

\section{Mathematics Subject Classification $\quad 70 \mathrm{H} 33 \cdot 70 \mathrm{H} 45$}

\section{Introduction}

The most important task in dynamics is to find the solution of the differential equations of motion. If one can find all of the integrals of the equations, the solution of the equations can be given. An integral is a conserved quantity, therefore, people make efforts to find all of the conserved quantities of mechanical system.

Newtonian mechanics provides us three conservation laws, i.e. the conservation of momentum, the conservation of moment of momentum and the conservation of mechanical energy, by means of the analysis of forces. The three conservation laws have very clear physical meaning. Lagrangian mechanics provides us two conservation laws, i.e. the conservation of generalized momentum and the conservation of generalized energy, by means of the analysis of the form of Lagrangian. The conservation of generalized momentum

F. X. Mei · H. B. Wu (凶) · Y. F. Zhang

School of Mathematics, Beijing Institute of Technology, No.5 Zhongguancun Nan Road, Haidian, Beijing 100081, China e-mail: huibinwu@bit.edu.cn may be a conservation of momentum, a conservation of moment of momentum, or neither. The physical meaning of the conservation law in Lagrangian mechanics is less clear than that in Newtonian mechanics, but the conserved quantities deduced by Lagrangian mechanics are more than that deduced by Newtonian mechanics. Since Noether published her well-known paper [1], the Noether symmetry method has become a modern method for seeking the conservation law of mechanical systems [2-18]. The physical meaning of the conservation law in the Noether symmetry method is less than that in Lagrangian mechanics, but the conserved quantities deduced by the Noether symmetry method are more than that deduced by Lagrangian mechanics.

The Noether symmetry is an invariance of the Hamilton action under the infinitesimal transformations of time and coordinates. Besides the Noether symmetry, there are two other important symmetries, i.e. the Lie symmetry and the form invariance. The Lie symmetry is a kind of invariance of the differential equations under the infinitesimal transformations of time and coordinates. The form invariance is a kind of invariance under which the transformed dynamical functions still satisfy the original differential equations of motion. A Noether symmetry can lead to a conserved quantity according to the Noether theory. A Lie symmetry or a form invariance can also lead to a conserved quantity under certain conditions. The conserved quantities deduced directly by the Noether symmetry, the Lie symmetry and the form invariance are called the Noether conserved quantity, the Hojman type conserved quantity and the new conserved quantity, respectively. The constrained mechanical systems in the review involves holonomic systems, nonholonomic systems and Birkhoffian systems.

The outline of this review is as follows: In Sect. 2, we study the Noether symmetry of constrained mechanical systems. In Sect. 3, we discuss the Lie symmetry of constrained 
mechanical systems. In Sect. 4, we present the form invariance of constrained mechanical systems. Section 5 deals with the Noether conserved quantity deduced by the Noether symmetry. Section 6 provides the Hojman type conserved quantity deduced by the Lie symmetry. In Sect. 7, we work at a new conserved quantity deduced by the form invariance. Section 8 goes into the Non-Noether conserved quantity guided by the Noether symmetry. Section 9 is devoted to the Noether conserved quantity and the new conserved quantity deduced by the Lie symmetry. In Sect. 10, we specialize the Noether conserved quantity and the Hojman type conserved quantity deduced by the form invariance. Finally, we propose some topics for future research on the study of the symmetry in Sect. 11.

\section{Noether symmetry}

The Noether symmetry is an invariance of the Hamilton action under the infinitesimal transformations of time and the coordinates.

\subsection{Noether symmetry of Lagrangian system}

The equations of motion of Lagrangian system have the form

$E_{S}(L)=0 \quad(s=1, \ldots, n)$,

where $L$ is the Lagrangian and $E_{S}$ the Euler operator

$E_{S}=\frac{\mathrm{d}}{\mathrm{d} t} \frac{\partial}{\partial \dot{q}_{s}}-\frac{\partial}{\partial q_{s}}$.

For the system (1), Arnold gave the Noether's theorem [19] as follows:

If the system $(M, L)$ admits the one-parameter group of diffeomorphisms $h^{s}: M \rightarrow M, s \in R$, then the Lagrangian system of equations corresponding to $L$ has a first integral $I: T M \rightarrow R$.

In local coordinates $q$ on $M$, the integral $I$ is written in the form

$I(q, \dot{q})=\left.\frac{\partial L}{\partial \dot{q}} \frac{\mathrm{d} h^{s}(q)}{\mathrm{d} s}\right|_{s=0}$.

José and Saletan wrote " if a Lagrangian is invariant under a family of transformations, its dynamical system possesses a constant of the motion, and that constant can be found from a knowledge of the Lagrangian and the transformation" [20].

Therefore, there are two comprehensions for the Noether symmetry. One comprehension is an invariance of the Hamilton action; another is an invariance of the Lagrangian. We all think that the first one is more reasonable.

Introducing the infinitesimal transformations of time and the generalized coordinates as

$t^{*}=t+\Delta t, \quad q_{s}^{*}\left(t^{*}\right)=q_{s}(t)+\Delta q_{s}$, or

$t^{*}=t+\varepsilon \xi_{0}(t, \boldsymbol{q}, \dot{\boldsymbol{q}})$,

$q_{s}^{*}\left(t^{*}\right)=q_{s}(t)+\varepsilon \xi_{s}(t, \boldsymbol{q}, \dot{\boldsymbol{q}})$,

where $\varepsilon$ is an infinitesimal parameter, and $\xi_{0}, \xi_{s}$ the infinitesimal generators, the invariance of the Hamilton action leads to the Noether identity

$L \dot{\xi}_{0}+X^{(1)}(L)+\dot{G}_{N}=0$,

where $G_{N}=G_{N}(t, \boldsymbol{q}, \dot{\boldsymbol{q}})$ is a gauge function and

$X^{(1)}=\xi_{0} \frac{\partial}{\partial t}+\xi_{s} \frac{\partial}{\partial q_{s}}+\left(\dot{\xi}_{s}-\dot{q}_{s} \dot{\xi}_{0}\right) \frac{\partial}{\partial \dot{q}_{s}}$.

If there exists a function $G_{N}$ such that the identity (6) is satisfied, then the corresponding symmetry is called a Noether symmetry. We will see that the conserved quantities can be found by using the Noether symmetry.

\subsection{Noether symmetry of general holonomic system}

The equations of motion of general holonomic system can be written in the form

$E_{s}(L)=Q_{s} \quad(s=1, \ldots, n)$,

where $Q_{s}=Q_{s}(t, \boldsymbol{q}, \dot{\boldsymbol{q}})$ are generalized forces. Under the infinitesimal transformations (5), the Noether symmetry of the system leads to the satisfaction of the following Noether identity

$L \dot{\xi}_{0}+X^{(1)}(L)+Q_{s}\left(\xi_{s}-\dot{q}_{s} \xi_{0}\right)+\dot{G}_{N}=0$.

Formula (9) is called the generalized Noether-Bessel-Hagen equation [8].

By means of the Noether symmetry, we can seek the conserved quantities of the general holonomic system.

\subsection{Noether symmetry of nonholonomic system}

The nonholonomic system is more complected than the holonomic system, e.g. see references [21-31].

Let the position of a mechanical system be determined by the $n$ generalized coordinates $q_{s}(s=1, \ldots, n)$ and its motion be subject to the $g$ ideal nonholonomic constraints of Chetaev's type

$f_{\beta}(t, \boldsymbol{q}, \dot{\boldsymbol{q}})=0 \quad(\beta=1, \ldots, g)$.

The differential equations of motion of the system can be written in the form [20,25,29-33]

$E_{s}(L)=Q_{s}+\lambda_{\beta} \frac{\partial f_{\beta}}{\partial \dot{q}_{s}}$,

where $\lambda_{\beta}$ are the constraint multipliers. Before integrating the differential equations, from Eqs. (10) and (11) one can solve 
the multipliers $\lambda_{\beta}$ as functions of $t, \boldsymbol{q}$ and $\dot{\boldsymbol{q}}$. So, Eqs. (11) have the form

$E_{s}(L)=Q_{s}+\Lambda_{s}$,

where

$\Lambda_{s}=\lambda_{\beta}(t, \boldsymbol{q}, \dot{\boldsymbol{q}}) \frac{\partial f_{\beta}}{\partial \dot{q}_{s}}$

express the nonholonomic constraint forces. Equations (12) are called the equations of holonomic system corresponding to the nonholonomic system (10), (11).

For the system (12), the Noether identity is

$$
L \dot{\xi}_{0}+X^{(1)}(L)+\left(Q_{s}+\Lambda_{s}\right)\left(\xi_{s}-\dot{q}_{s} \xi_{0}\right)+\dot{G}_{N}=0 .
$$

The restriction of nonholonomic constraints (10) on the generators has the form [13]

$\frac{\partial f_{\beta}}{\partial \dot{q}_{s}}\left(\xi_{s}-\dot{q}_{s} \xi_{0}\right)=0$.

If the generators $\xi_{0}, \xi_{s}$ and the gauge function $G_{N}$ satisfy Eqs. (14) and (15), then the symmetry is a Noether one of the nonholonomic system. If they satisfy only Eq. (14), then the symmetry is a Noether symmetry of the corresponding holonomic system (12).

Considering Eq. (15), the identity (14) can be simplified as

$L \dot{\xi}_{0}+X^{(1)}(L)+Q_{s}\left(\xi_{s}-\dot{q}_{s} \xi_{0}\right)+\dot{G}_{N}=0$.

The complexity of the Noether symmetry of the nonholonomic system is to have the restriction of nonholonomic constraints on the generators.

The conserved quantities can be obtained by using the Noether symmetry.

\subsection{Noether symmetry of Birkhoffian system}

The Birkhoffian system is a kind of constrained mechanical systems. The Birkhoff equations have the form [34]

$\Omega_{\mu \nu} \dot{a}^{\nu}-\frac{\partial B}{\partial a^{\mu}}-\frac{\partial R_{\mu}}{\partial t}=0 \quad(\mu, v=1, \ldots, 2 n)$,

where

$\Omega_{\mu \nu}=\frac{\partial R_{v}}{\partial a^{\mu}}-\frac{\partial R_{\mu}}{\partial a^{v}}$,

and $B=B(t, a)$ is the Birkhoffian and $R_{\mu}=R_{\mu}(t, a)$ the Birkhoff's functions.

Introducing the infinitesimal transformations as

$t^{*}=t+\varepsilon \xi_{0}(t, \boldsymbol{a}), \quad a^{\mu *}=a^{\mu}+\varepsilon \xi_{\mu}(t, \boldsymbol{a})$

for the system (17), the Noether identity has the form [14]

$$
\begin{aligned}
& \left(\frac{\partial R_{\mu}}{\partial t} \dot{a}^{\mu}-\frac{\partial B}{\partial t}\right) \xi_{0}+\left(\frac{\partial R_{v}}{\partial a^{\mu}} \dot{a}^{\nu}-\frac{\partial B}{\partial a^{\mu}}\right) \xi_{\mu}-B \dot{\xi}_{0} \\
& \quad+R_{\mu} \dot{\xi}_{\mu}+\dot{G}_{N}=0,
\end{aligned}
$$

where $G_{N}=G_{N}(t, \boldsymbol{a})$ is the gauge function.
The Noether symmetry of Birkhoffian system can lead to the conserved quantities.

The study on Birkhoffian mechanics can be seen in reference [35].

\section{Lie symmetry}

The Lie symmetry is an invariance of the differential equations of motion under the infinitesimal transformations. On the part of mathematics, the monographs are very important, e.g. see references [36-39]. There have been some important results on the study of the Lie symmetry of mechanical systems [40-48].

\subsection{Lie symmetry of Lagrangian system}

Suppose that the system (1) is nonsingular, i.e.

$$
\operatorname{det}\left(\frac{\partial^{2} L}{\partial \dot{q}_{s} \partial \dot{q}_{k}}\right) \neq 0 .
$$

Then Eq. (1) can be written as

$\ddot{q}_{s}=\alpha_{s}(t, \boldsymbol{q}, \dot{\boldsymbol{q}})$.

Under the infinitesimal transformations (5), the determining equations of the Lie symmetry of Eq. (22) have the form

$\ddot{q}_{s}-\dot{q}_{s} \ddot{\xi}_{0}-2 \dot{\xi}_{0} \alpha_{s}=X^{(1)}\left(\alpha_{s}\right)$.

The Lie symmetry can lead to the conserved quantities under certain conditions. But it is not easy that one find the generators $\xi_{0}, \xi_{s}$ from Eq. (23), because symmetry methods for differential equations, originally developed by Sophus Lie, are highly algorithmic [37].

For the Lagrangian system, all of the Noether symmetries are also Lie symmetries $[49,50]$.

\subsection{Lie symmetry of general holonomic system}

Let Eq. (8) have the form

$\ddot{q}_{s}=\beta_{s}(t, \boldsymbol{q}, \dot{\boldsymbol{q}})$.

The determining equations of the Lie symmetry of Eq. (24) have the form

$\ddot{\xi}_{s}-\dot{q}_{s} \ddot{\xi}_{0}-2 \dot{\xi}_{0} \beta_{s}=X^{(1)}\left(\beta_{s}\right)$.

If the infinitesimal generators $\xi_{0}, \xi_{s}$ satisfy Eq. (25), then the Lie symmetry can lead to the conserved quantities under certain conditions.

For the general holonomic system, a Noether symmetry is a Lie symmetry under certain conditions [50]. 
3.3 Lie symmetry of nonholonomic system

From Eq. (12), we can solve all of the generalized accelerations as

$\ddot{q}_{s}=\gamma_{s}(t, \boldsymbol{q}, \dot{\boldsymbol{q}})$.

Their determining equations of the Lie symmetry are

$\ddot{\xi}_{s}-\dot{q}_{s} \ddot{\xi}_{0}-2 \dot{\xi}_{0} \gamma_{s}=X^{(1)}\left(\gamma_{s}\right)$.

The restriction equations of nonholonomic constraints (10) have the form

$X^{(1)}\left(f_{\beta}(t, \boldsymbol{q}, \dot{\boldsymbol{q}})\right)=0$.

If the generators $\xi_{0}, \xi_{s}$ satisfy the determining equations (27) and the restriction equations (28), then the symmetry is a Lie symmetry of the nonholonomic system. If the generators $\xi_{0}$, $\xi_{s}$ satisfy only the determining equations (27), then the symmetry is a Lie symmetry of the corresponding holonomic system. The complexity of the Lie symmetry of the nonholonomic system is to have the restriction conditions (28) of the nonholonomic constraint to the infinitesimal generators.

\subsection{Lie symmetry of Birkhoffian system}

Suppose that

$\operatorname{det}\left(\Omega_{\mu \nu}\right) \neq 0$.

Then from Eq. (17), we can solve $\dot{a}^{\mu}$ as

$\dot{a}^{\mu}-\Omega^{\mu v}\left(\frac{\partial B}{\partial a^{v}}+\frac{\partial R_{v}}{\partial t}\right)=0$.

Under the infinitesimal transformations (19), the determining equations of the Lie symmetry of Eq. (30) have the form

$\dot{\xi}_{\mu}-\Omega^{\mu \nu}\left(\frac{\partial B}{\partial a^{\nu}}+\frac{\partial R_{v}}{\partial t}\right) \dot{\xi}_{0}=X^{(0)}\left\{\Omega^{\mu \nu}\left(\frac{\partial B}{\partial a^{\nu}}+\frac{\partial R_{v}}{\partial t}\right)\right\}$,

where

$X^{(0)}=\xi_{0} \frac{\partial}{\partial t}+\xi_{\mu} \frac{\partial}{\partial a^{\mu}}$.

If the infinitesimal generators $\xi_{0}, \xi_{s}$ satisfy the determining equations (31), then the symmetry is a Lie symmetry of the Birkhoffian system.

\section{Form invariance}

There are dynamical functions in the differential equations of motion of mechanical systems, e.g. the Lagrangian, the generalized forces, the generalized constraint forces in the holonomic and nonholonomic systems, the Birkhoffian, the
Birkhoff functions in the Birkhoffian system. If the transformed dynamical functions still satisfy the original differential equations of motion, then the symmetry is called a form invariance. There have been some important results on the study of the form invariance [51-61].

\subsection{Form invariance of Lagrangian system}

After the transformations (5), the Lagrangian $L(t, \boldsymbol{q}, \dot{\boldsymbol{q}})$ becomes $L\left(t^{*}, \boldsymbol{q}^{*}, \frac{\mathrm{d} \boldsymbol{q}^{*}}{\mathrm{~d} t^{*}}\right)$, we have

$L\left(t^{*}, \boldsymbol{q}^{*}, \frac{\mathrm{d} \boldsymbol{q}^{*}}{\mathrm{~d} t^{*}}\right)=L(t, \boldsymbol{q}, \dot{\boldsymbol{q}})+\varepsilon X^{(1)}(L)+0\left(\varepsilon^{2}\right)$.

Substituting (33) into Eq. (1) and neglecting $\varepsilon^{2}$ terms and the higher terms, we obtain

$E_{S}\left\{X^{(1)}(L)\right\}=0$.

If the generators $\xi_{0}, \xi_{s}$ satisfy the Eq. (34), then the symmetry is a form invariance of the Lagrangian system. Equation (34) are called the criterion equations of the form invariance of the Lagrangian system. The form invariance is different from the Noether symmetry and the Lie symmetry.

\subsection{Form invariance of general holonomic system}

After the transformations (5), the Lagrangian $L(t, \boldsymbol{q}, \dot{\boldsymbol{q}})$ and the generalized forces $Q_{s}(t, \boldsymbol{q}, \dot{\boldsymbol{q}})$ become respectively $L\left(t^{*}, \boldsymbol{q}^{*}, \frac{\mathrm{d} \boldsymbol{q}^{*}}{\mathrm{~d} t^{*}}\right)$ and $Q_{s}\left(t^{*}, \boldsymbol{q}^{*}, \frac{\mathrm{d} \boldsymbol{q}^{*}}{\mathrm{~d} t^{*}}\right)$, and we have

$$
\begin{aligned}
L\left(t^{*}, \boldsymbol{q}^{*}, \frac{\mathrm{d} \boldsymbol{q}^{*}}{\mathrm{~d} t^{*}}\right) & =L(t, \boldsymbol{q}, \dot{\boldsymbol{q}})+\varepsilon X^{(1)}(L)+0\left(\varepsilon^{2}\right), \\
Q_{s}\left(t^{*}, \boldsymbol{q}^{*}, \frac{\mathrm{d} \boldsymbol{q}^{*}}{\mathrm{~d} t^{*}}\right) & =Q_{s}(t, \boldsymbol{q}, \dot{\boldsymbol{q}})+\varepsilon X^{(1)}\left(Q_{s}\right)+0\left(\varepsilon^{2}\right) .
\end{aligned}
$$

Substituting (35) into Eq. (8) and neglecting $\varepsilon^{2}$ terms and the higher terms, we obtain

$E_{s}\left\{X^{(1)}(L)\right\}=X^{(1)}\left(Q_{s}\right)$.

If the generators $\xi_{0}, \xi_{s}$ satisfy the Eq. (36), then the symmetry is a form invariance of the general holonomic system. Equation (36) are called the criterion equations of the form invariance of the general holonomic system.

\subsection{Form invariance of nonholonomic system}

After the transformations (5), the Lagrangian $L(t, \boldsymbol{q}, \dot{\boldsymbol{q}})$, the generalized forces $Q_{s}(t, \boldsymbol{q}, \dot{\boldsymbol{q}})$ and the generalized constraint forces $\Lambda_{s}(t, \boldsymbol{q}, \dot{\boldsymbol{q}})$ become respectively $L\left(t^{*}, \boldsymbol{q}^{*}, \frac{\mathrm{d} \boldsymbol{q}^{*}}{\mathrm{~d} t^{*}}\right)$, $Q_{s}\left(t^{*}, \boldsymbol{q}^{*}, \frac{\mathrm{d} \boldsymbol{q}^{*}}{\mathrm{~d} t^{*}}\right)$ and $\Lambda_{s}\left(t^{*}, \boldsymbol{q}^{*}, \frac{\mathrm{d} \boldsymbol{q}^{*}}{\mathrm{~d} t^{*}}\right)$, and we have 
$L\left(t^{*}, \boldsymbol{q}^{*}, \frac{\mathrm{d} \boldsymbol{q}^{*}}{\mathrm{~d} t^{*}}\right)=L(t, \boldsymbol{q}, \dot{\boldsymbol{q}})+\varepsilon X^{(1)}(L)+0\left(\varepsilon^{2}\right)$,

$Q_{s}\left(t^{*}, \boldsymbol{q}^{*}, \frac{\mathrm{d} \boldsymbol{q}^{*}}{\mathrm{~d} t^{*}}\right)=Q_{s}(t, \boldsymbol{q}, \dot{\boldsymbol{q}})+\varepsilon X^{(1)}\left(Q_{s}\right)+0\left(\varepsilon^{2}\right)$,

$\Lambda_{s}\left(t^{*}, \boldsymbol{q}^{*}, \frac{\mathrm{d} \boldsymbol{q}^{*}}{\mathrm{~d} t^{*}}\right)=\Lambda_{s}(t, \boldsymbol{q}, \dot{\boldsymbol{q}})+\varepsilon X^{(1)}\left(\Lambda_{s}\right)+0\left(\varepsilon^{2}\right)$.

Substituting (37) into Eq. (12) and neglecting $\varepsilon^{2}$ terms and the higher terms, we obtain

$E_{S}\left\{X^{(1)}(L)\right\}=X^{(1)}\left(Q_{s}\right)+X^{(1)}\left(\Lambda_{S}\right)$

If the generators $\xi_{0}, \xi_{s}$ satisfy the Eq. (38), then the symmetry is a form invariance of the corresponding holonomic system (12).

After the transformations (5), the constraint functions $f_{\beta}(t, \boldsymbol{q}, \dot{\boldsymbol{q}})$ become $f_{\beta}^{*}\left(t^{*}, \boldsymbol{q}^{*}, \frac{\mathrm{d} \boldsymbol{q}^{*}}{\mathrm{~d} t^{*}}\right)$, and we have

$f_{\beta}^{*}=f_{\beta}+\varepsilon X^{(1)}\left(f_{\beta}\right)+0\left(\varepsilon^{2}\right)$.

Substituting (39) into the constraint equations (10) and neglecting $\varepsilon^{2}$ terms and the higher terms, we obtain

$X^{(1)}\left(f_{\beta}\right)=0$.

If the generators $\xi_{0}, \xi_{s}$ satisfy the criterion equations (38) and the restriction equations (40), then the symmetry is a form invariance of the nonholonomic system. The complexity of the form invariance of the nonholonomic system is to have the restriction (40) of the nonholonomic constraints on the generators.

\subsection{Form invariance of Birkhoffian system}

After the transformations (19), the Birkhoffian $B(t, \boldsymbol{a})$ and the Birkhoff functions $R_{\mu}(t, \boldsymbol{a})$ become respectively $B\left(t^{*}, \boldsymbol{a}^{*}\right)$ and $R_{\mu}\left(t^{*}, \boldsymbol{a}^{*}\right)$, and we have

$$
\begin{aligned}
B\left(t^{*}, \boldsymbol{a}^{*}\right) & =B(t, \boldsymbol{a})+\varepsilon X^{(0)}(B)+0\left(\varepsilon^{2}\right), \\
R_{\mu}\left(t^{*}, \boldsymbol{a}^{*}\right) & =R_{\mu}(t, \boldsymbol{a})+\varepsilon X^{(0)}\left(R_{\mu}\right)+0\left(\varepsilon^{2}\right) .
\end{aligned}
$$

Substituting (41) into Eq. (17) and neglecting $\varepsilon^{2}$ terms and the higher terms, we obtain

$$
\begin{aligned}
& \left\{\frac{\partial}{\partial a^{\mu}} X^{(0)}\left(R_{v}\right)-\frac{\partial}{\partial a^{v}} X^{(0)}\left(R_{\mu}\right)\right\} \dot{a}^{v}-\frac{\partial}{\partial a^{\mu}} X^{(0)}(B) \\
& -\frac{\partial}{\partial t} X^{(0)}\left(R_{\mu}\right)=0
\end{aligned}
$$

If the generators $\xi_{0}, \xi_{\mu}$ satisfy the criterion equations (42), then the symmetry is a form invariance of the Birkhoffian system.

\section{Noether conserved quantities deduced by Noether symmetry}

In Sects. 5-10, we will present three kinds of conserved quantities deduced by using three kinds of symmetries for the constrained mechanical systems and give some practical examples to illustrate the application of the results.

The Noether symmetry can lead directly to the Noether conserved quantity by using the Noether theorem.

\subsection{Lagrangian system}

Proposition 5.1 For the Lagrangian system (1), if the infinitesimal generators $\xi_{0}, \xi_{s}$ and the gauge function $G_{N}$ satisfy the Noether identity (6), then the Noether symmetry will lead to the Noether conserved quantity

$I_{N}=L \xi_{0}+\frac{\partial L}{\partial \dot{q}_{s}}\left(\xi_{s}-\dot{q}_{s} \xi_{0}\right)+G_{N}=$ const.

This is the Noether theorem of the Lagrangian system.

Example 5.1 The Lagrangian of a harmonic oscillator system with two-degree-of-freedom is [62]

$L=\frac{1}{2} m\left(\dot{q}_{1}^{2}+\dot{q}_{2}^{2}\right)-\frac{1}{2} k\left(q_{1}^{2}+q_{2}^{2}\right)$

where $m, k$ are constants.

The Noether identity (6) gives

$$
\begin{aligned}
& \left\{\frac{1}{2} m\left(\dot{q}_{1}^{2}+\dot{q}_{2}^{2}\right)-\frac{1}{2} k\left(q_{1}^{2}+q_{2}^{2}\right)\right\} \dot{\xi}_{0}+m \dot{q}_{1}\left(\dot{\xi}_{1}-\dot{q}_{1} \dot{\xi}_{0}\right) \\
& \quad+m \dot{q}_{2}\left(\dot{\xi}_{2}-\dot{q}_{2} \dot{\xi}_{0}\right)-k q_{1} \xi_{1}-k q_{2} \xi_{2}+\dot{G}_{N}=0 .
\end{aligned}
$$

It has the following solutions:

$\xi_{0}=-1, \quad \xi_{1}=\xi_{2}=0, \quad G_{N}=0$,

$\xi_{0}=0, \quad \xi_{1}=-q_{2}, \quad \xi_{2}=q_{1}, \quad G_{N}=0$,

$\xi_{0}=-1, \quad \xi_{1}=0, \quad \xi_{2}=-\dot{q}_{2}, \quad G_{N}=\frac{1}{2} m \dot{q}_{2}^{2}-\frac{1}{2} k q_{2}^{2}$,

$\xi_{0}=-1, \quad \xi_{1}=-\dot{q}_{1}, \quad \xi_{2}=0, \quad G_{N}=\frac{1}{2} m \dot{q}_{1}^{2}-\frac{1}{2} k q_{1}^{2}$,

$\xi_{0}=0, \quad \xi_{1}=\dot{q}_{1}+\frac{1}{2} \dot{q}_{2}, \quad \xi_{2}=\dot{q}_{2}+\frac{1}{2} \dot{q}_{1}$,

$G_{N}=-L+\frac{1}{2} k q_{1} q_{2}-\frac{1}{2} m \dot{q}_{1} \dot{q}_{2}$

By using Proposition 5.1, we obtain the following Noether conserved quantities: 
$I_{N_{1}}=\frac{1}{2} m\left(\dot{q}_{1}^{2}+\dot{q}_{2}^{2}\right)+\frac{1}{2} k\left(q_{1}^{2}+q_{2}^{2}\right)=$ const.,

$I_{N_{2}}=m\left(q_{1} \dot{q}_{2}-q_{2} \dot{q}_{1}\right)=$ const.

$I_{N_{3}}=\frac{1}{2} m \dot{q}_{1}^{2}+\frac{1}{2} k q_{1}^{2}=$ const.,

$I_{N_{4}}=\frac{1}{2} m \dot{q}_{2}^{2}+\frac{1}{2} k q_{2}^{2}=$ const.,

$I_{N_{5}}=\frac{1}{2} m \dot{q}_{1} \dot{q}_{2}+\frac{1}{2} k q_{1} q_{2}=$ const.

These five integrals are not independent, because [62]

$I_{N_{1}}=I_{N_{3}}+I_{N_{4}}$

$I_{N_{2}}=2\left\{\frac{m}{k}\left(I_{N_{3}} I_{N_{4}}-I_{N_{5}}^{2}\right)\right\}^{1 / 2}$.

The integral $I_{N_{1}}$ is the integral of energy, the integral $I_{N_{2}}$ is the integral of momentum moment, the integrals $I_{N_{3}}$ and $I_{N_{4}}$ are the local integrals of energy. The Lagrangian mechanics only provides us the integral $I_{N_{1}}$.

Example 5.2 The Emden equation is

$\ddot{q}+\frac{2}{t} \dot{q}+q^{5}=0$.

This is a Lagrangian system whose Lagrangian has the form

$L=t^{2}\left(\frac{1}{2} \dot{q}^{2}-\frac{1}{6} q^{6}\right)$.

The Noether identity (6) gives

$L \dot{\xi}_{0}+t\left(\dot{q}^{2}-\frac{1}{3} q^{6}\right) \xi_{0}+t^{2} \dot{q}\left(\dot{\xi}-\dot{q} \dot{\xi}_{0}\right)-t^{2} q^{5} \xi+\dot{G}_{N}=0$.

It has the solution

$\xi_{0}=-2 t, \quad \xi=q, \quad G_{N}=0$.

The Noether conserved quantity (43) gives

$I_{N}=t^{3} \dot{q}^{2}+t^{2} q \dot{q}+\frac{1}{3} t^{3} q^{6}=$ const.

\subsection{General holonomic system}

Proposition 5.2 For the general holonomic system (8), if the infinitesimal generators $\xi_{0}, \xi_{s}$ and the gauge function $G_{N}$ satisfy the Noether identity (9), then the Noether symmetry will lead to the Noether conserved quantity

$I_{N}=L \xi_{0}+\frac{\partial L}{\partial \dot{q}_{s}}\left(\xi_{s}-\dot{q}_{s} \xi_{0}\right)+G_{N}=$ const.

This is the Noether theorem of the general holonomic system.

Example 5.3 The differential equation model of the armament race can be written in the form

$\ddot{q}+(\alpha+\beta) \dot{q}+(\alpha \beta-k l) q=0$, where $\alpha, \beta, k$ and $l$ are constants. It is a particular case of the Lanchester model [63]. The equation can be considered as the equation of a holonomic system whose Lagrangian is

$L=\frac{1}{2} \dot{q}^{2}-\frac{1}{2}(\alpha \beta-k l) q^{2}$,

and the generalized force is

$Q=-(\alpha+\beta) \dot{q}$.

The Noether identity (9) gives

$$
\begin{aligned}
& \left\{\frac{1}{2} \dot{q}^{2}-\frac{1}{2}(\alpha \beta-k l) q^{2}\right\} \dot{\xi}_{0}+\dot{q}\left(\dot{\xi}-\dot{q} \dot{\xi}_{0}\right) \\
& \quad-(\alpha \beta-k l) q \xi-(\alpha+\beta) \dot{q}\left(\xi-\dot{q} \xi_{0}\right)+\dot{G}_{N}=0 .
\end{aligned}
$$

which has the solution

$\xi_{0}=0, \quad \xi=\exp A t, \quad G_{N}=q(\alpha+\beta-A) \exp A t$,

where the constant $A$ is the solution of the following algebraic equation

$A^{2}-(\alpha+\beta) A+\alpha \beta-k l=0$.

The Noether conserved quantity (44) gives

$I_{N}=\{\dot{q}+(\alpha+\beta-A)\} \exp A t=$ const.

Example 5.4 The differential equation is

$\ddot{q}+k n q^{n-1}=-\mu\left(a \dot{q}+b \dot{q}^{2}\right)$,

where $n$ is a positive integer, $a, b$ and $k$ are constants and $\mu \ll 1$ [8].

The equation can be considered as the equation of a holonomic system whose Lagrangian is

$L=\frac{1}{2} \dot{q}^{2}-k q^{n}$

and the generalized force is

$Q=-\mu\left(a \dot{q}+b \dot{q}^{2}\right)$.

There is no exact constant of motion in the example. In the following, we seek the approximate constant of motion of the system by using the Noether theorem. Let

$\xi_{0}=\left(\xi_{0}\right)_{0}+\mu\left(\xi_{0}\right)_{1}+\cdots$,

$\xi=(\xi)_{0}+\mu(\xi)_{1}+\cdots$,

$G_{N}=\left(G_{N}\right)_{0}+\mu\left(G_{N}\right)_{1}+\cdots$.

Substituting them into the Noether identity (9), we obtain

$$
\begin{aligned}
& -k n q^{n-1}\left\{(\xi)_{0}+\mu(\xi)_{1}+\cdots\right\}+\dot{q}\left\{(\dot{\xi})_{0}+\mu(\dot{\xi})_{1}+\cdots\right\} \\
& +\left(-\frac{1}{2} \dot{q}^{2}-k q^{n}\right)\left\{\left(\dot{\xi}_{0}\right)_{0}+\mu\left(\dot{\xi}_{0}\right)_{1}+\cdots\right\} \\
& -\mu\left(a \dot{q}+b \dot{q}^{2}\right)\left\{(\xi)_{0}+\mu(\xi)_{1}+\cdots-\dot{q}\left(\xi_{0}\right)_{0}\right. \\
& \left.-\mu \dot{q}\left(\xi_{0}\right)_{1}-\cdots\right\} \\
& +\left(\dot{G}_{N}\right)_{0}+\mu\left(\dot{G}_{N}\right)_{1}+\cdots=0 .
\end{aligned}
$$


Separating the terms containing $\mu$ and the terms not containing $\mu$, we obtain

$$
\begin{aligned}
& -k n q^{n-1}(\xi)_{0}+\dot{q}(\dot{\xi})_{0}-\left(\frac{1}{2} \dot{q}^{2}+k q^{n}\right)\left(\dot{\xi}_{0}\right)_{0}+\left(\dot{G}_{N}\right)_{0}=0, \\
& -k n q^{n-1}(\xi)_{1}+\dot{q}(\dot{\xi})_{1}-\left(\frac{1}{2} \dot{q}^{2}+k q^{n}\right)\left(\dot{\xi}_{0}\right)_{1} \\
& -\left(a \dot{q}+b \dot{q}^{2}\right)\left[(\xi)_{0}-\dot{q}\left(\xi_{0}\right)_{0}\right]+\left(\dot{G}_{N}\right)_{1}=0 .
\end{aligned}
$$

which have the solution

$$
\begin{aligned}
& \left(\xi_{0}\right)_{0}=1, \quad(\xi)_{0}=0, \quad\left(G_{N}\right)_{0}=0, \\
& \left(\xi_{0}\right)_{1}=2 b q+\frac{2 a n t}{2+n}, \quad(\xi)_{1}=-\frac{2 a q}{2+n}, \quad\left(G_{N}\right)_{1}=2 b k \frac{q^{n+1}}{1+n} .
\end{aligned}
$$

The Noether conserved quantity (44) gives

$$
\begin{aligned}
I_{N}= & \left(\frac{1}{2} \dot{q}^{2}-k q^{n}\right)\left\{1+2 \mu\left(b q+\frac{a n t}{2+n}\right)\right\} \\
& +\dot{q}\left\{-\mu \frac{2 a q}{2+n}-\dot{q}\left[1+2 \mu\left(b q+\frac{a n t}{2+n}\right)\right]\right\} \\
& +2 \mu b k \frac{q^{n+1}}{1+n},
\end{aligned}
$$

where the $\mu^{2}$ terms and the higher terms are neglected, therefore, $I_{N}$ is not a constant of motion. But we have

$\frac{\mathrm{d} I_{N}}{\mathrm{~d} t}=\mu^{2}(\cdots)+\cdots \approx 0$,

and $I_{N}$ is a generalized adiabatic invariant [8].

\subsection{Nonholonomic system}

Proposition 5.3 For the nonholonomic system (10), (11), if the infinitesimal generators $\xi_{0}, \xi_{s}$ and the gauge function $G_{N}$ satisfy Eqs. (14) and (15), then the Noether symmetry will lead to the Noether conserved quantity (43) [32].

Example 5.5 The Lagrangian and the constraint equation of a nonholonomic system are respectively

$$
\begin{aligned}
& L=\frac{1}{2} m\left(\dot{q}_{1}^{2}+\dot{q}_{2}^{2}+\dot{q}_{3}^{2}\right)-m g q_{3}, \\
& f=\dot{q}_{3}-\left(\dot{q}_{1}^{2}+\dot{q}_{2}^{2}\right)^{1 / 2}=0 .
\end{aligned}
$$

This is the celebrated Appell-Hamel problem [22, 25,26].

The Noether identity (16) and Eq. (15) give respectively

$$
\begin{aligned}
& L \dot{\xi}_{0}+m \dot{q}_{1}\left(\dot{\xi}_{1}-\dot{q}_{1} \dot{\xi}_{0}\right)+m \dot{q}_{2}\left(\dot{\xi}_{2}-\dot{q}_{2} \dot{\xi}_{0}\right) \\
& \quad+m \dot{q}_{3}\left(\dot{\xi}_{3}-\dot{q}_{3} \dot{\xi}_{0}\right)-m g \xi_{3}+\dot{G}_{N}=0, \\
& \xi_{3}-\dot{q}_{3} \xi_{0}-\left(\dot{q}_{1}^{2}+\dot{q}_{2}^{2}\right)^{-1 / 2} \\
& \quad \times\left\{\dot{q}_{1}\left(\xi_{1}-\dot{q}_{1} \xi_{0}\right)+\dot{q}_{2}\left(\xi_{2}-\dot{q}_{2} \xi_{0}\right)\right\}=0 .
\end{aligned}
$$

They have the following solutions:

$$
\begin{aligned}
& \xi_{0}=-1, \quad \xi_{1}=\xi_{2}=\xi_{3}=0, \quad G_{N}=0, \\
& \xi_{0}=0, \quad \xi_{1}=-\frac{1}{\dot{q}_{2}}, \quad \xi_{2}=\frac{\dot{q}_{1}}{\dot{q}_{2}^{2}}, \quad \xi_{3}=0, \quad G_{N}=-m \frac{\dot{q}_{1}}{\dot{q}_{2}}, \\
& \xi_{0}=0, \quad \xi_{1}=0, \quad \xi_{2}=\dot{q}_{3}, \quad \xi_{3}=\dot{q}_{2}, \\
& G_{N}=m g q_{2}-m \dot{q}_{2} \dot{q}_{3}, \\
& \xi_{0}=0, \quad \xi_{1}=\dot{q}_{3}, \quad \xi_{2}=0, \quad \xi_{3}=\dot{q}_{1}, \\
& G_{N}=m g q_{1}-m \dot{q}_{1} \dot{q}_{3} .
\end{aligned}
$$

The corresponding Noether conserved quantities are respectively

$$
\begin{aligned}
& I_{N_{1}}=\frac{1}{2} m\left(\dot{q}_{1}^{2}+\dot{q}_{2}^{2}+\dot{q}_{3}^{2}\right)+m g q_{3}=\text { const., } \\
& I_{N_{2}}=-m \frac{\dot{q}_{1}}{\dot{q}_{3}}=\text { const., } \\
& I_{N_{3}}=m \dot{q}_{2} \dot{q}_{3}+m g q_{2}=\text { const., } \\
& I_{N_{4}}=m \dot{q}_{1} \dot{q}_{3}+m g q_{1}=\text { const. }
\end{aligned}
$$

Example 5.6 The Lagrangian and the constraint equation of a nonholonomic system are respectively

$L=\frac{1}{2}\left(\dot{q}_{1}^{2}+\dot{q}_{2}^{2}\right)$,

$f=\dot{q}_{1}+b t \dot{q}_{2}-b q_{2}+t=0$,

where $b$ is a constant [25].

Equation (12) give

$$
\ddot{q}_{1}=-\frac{1}{1+b^{2} t^{2}}, \quad \ddot{q}_{2}=-\frac{b t}{1+b^{2} t^{2}} .
$$

The Noether identity (14) becomes

$$
\begin{aligned}
& \frac{1}{2}\left(\dot{q}_{1}^{2}+\dot{q}_{2}^{2}\right) \dot{\xi}_{0}+\dot{q}_{1}\left(\dot{\xi}_{1}-\dot{q}_{1} \dot{\xi}_{0}\right)+\dot{q}_{2}\left(\dot{\xi}_{2}-\dot{q}_{2} \dot{\xi}_{0}\right) \\
& -\frac{1}{1+b^{2} t^{2}}\left(\xi_{1}-\dot{q}_{1} \xi_{0}\right)-\frac{b t}{1+b^{2} t^{2}}\left(\xi_{2}-\dot{q}_{2} \xi_{0}\right)+\dot{G}_{N}=0 .
\end{aligned}
$$

Equation (15) gives

$$
\xi_{1}-\dot{q}_{1} \xi_{0}+b t\left(\xi_{2}-\dot{q}_{2} \xi_{0}\right)=0 .
$$

The above two equations have the solution

$\xi_{0}=0, \quad \xi_{1}=b t, \quad \xi_{2}=-1, \quad G_{N}=-b q_{1}$.

Thus, the Noether conserved quantity (43) gives

$I_{N}=\dot{q}_{1} b t-\dot{q}_{2}-b q_{1}=$ const.

\subsection{Birkhoffian system}

Proposition $5.4[14,34]$ For the Birkhoffian system (17), if the generators of infinitesimal transformations $\xi_{0}, \xi_{\mu}$ and the gauge function $G_{N}$ satisfy the Noether identity (20), then the Noether symmetry will lead to the Noether conserved quantity

$I_{N}=R_{\mu} \xi_{\mu}-B \xi_{0}+G_{N}=$ const. 
Example 5.7 [34] The Birkhoffian and the Birkhoff's functions of a system are respectively

$B=\frac{1}{2}\left\{\left(a^{3}\right)^{2}+2 a^{2} a^{3}-\left(a^{4}\right)^{2}\right\}$,

$R_{1}=a^{2}+a^{3}, \quad R_{2}=0, \quad R_{3}=a^{4}, \quad R_{4}=0$.

The Noether identity (20) gives

$$
\begin{aligned}
& \left(\dot{a}^{1}-a^{3}\right) \xi_{2}+\left(\dot{a}^{1}-a^{2}-a^{3}\right) \xi_{3}+\left(\dot{a}^{3}+a^{4}\right) \xi_{4}-B \dot{\xi}_{0} \\
& +\left(a^{2}+a^{3}\right) \dot{\xi}_{1}+a^{4} \dot{\xi}_{3}+\dot{G}_{N}=0 .
\end{aligned}
$$

It has the following solutions:

$\xi_{0}=0, \quad \xi_{1}=\cos t, \quad \xi_{2}=\sin t, \quad \xi_{3}=-\sin t, \quad \xi_{4}=\cos t$, $G_{N}=-a^{3} \cos t$

$\xi_{0}=0, \quad \xi_{1}=\sin t, \quad \xi_{2}=-\cos t, \quad \xi_{3}=\cos t, \quad \xi_{4}=\sin t$,

$G_{N}=-a^{3} \sin t$,

$\xi_{0}=0, \quad \xi_{1}=1, \quad \xi_{2}=\xi_{3}=\xi_{4}=0, \quad G_{N}=0$,

$\xi_{0}=0, \quad \xi_{1}=-t, \quad \xi_{2}=0, \quad \xi_{3}=-1, \quad \xi_{4}=0, \quad G_{N}=a^{1}$.

The Noether conserved quantity (45) gives respectively

$I_{N_{1}}=a^{2} \cos t-a^{4} \sin t=C_{1}$,

$I_{N_{2}}=a^{2} \sin t+a^{4} \cos t=C_{2}$,

$I_{N_{3}}=a^{2}+a^{3}=C_{3}$,

$I_{N_{4}}=a^{1}-a^{4}-\left(a^{2}+a^{3}\right) t=C_{4}$.

from which we obtain the solution as

$a^{1}=-C_{1} \sin t+C_{2} \cos t+C_{3} t+C_{4}$,

$a^{2}=C_{1} \cos t+C_{2} \sin t$,

$a^{3}=-C_{1} \cos t-C_{2} \sin t+C_{3}$,

$a^{4}=-C_{1} \sin t+C_{2} \cos t$.

\section{Hojman type conserved quantities deduced by Lie symmetry}

Hojman gave a conserved quantity by using the Lie symmetry under the infinitesimal transformations where time is not changed [43]. Clearly, this result is applicable to the constrained mechanical systems.

\subsection{Lagrangian system}

Choosing the infinitesimal transformations as

$t^{*}=t, \quad q_{s}^{*}\left(t^{*}\right)=q_{s}(t)+\varepsilon \xi_{s}(t, \boldsymbol{q}, \dot{\boldsymbol{q}})$,

the determining equations (23) of the Lie symmetry become

$\frac{\overline{\mathrm{d}}}{\mathrm{d} t} \frac{\overline{\mathrm{d}}}{\mathrm{d} t} \xi_{s}=\frac{\partial \alpha_{s}}{\partial q_{k}} \xi_{k}+\frac{\partial \alpha_{s}}{\partial \dot{q}_{k}} \frac{\overline{\mathrm{d}}}{\mathrm{d} t} \xi_{k}$, where

$\frac{\overline{\mathrm{d}}}{\mathrm{d} t}=\frac{\partial}{\partial t}+\dot{q}_{s} \frac{\partial}{\partial q_{s}}+\alpha_{s} \frac{\partial}{\partial \dot{q}_{s}}$.

Proposition 6.1 For the Lagrangian system (22), if the infinitesimal generators $\xi_{s}$ satisfy the determining equations (47) and there exists a function $\mu=\mu(t, \boldsymbol{q}, \dot{\boldsymbol{q}})$ such that

$\frac{\partial \alpha_{s}}{\partial \dot{q}_{s}}+\frac{\overline{\mathrm{d}}}{\mathrm{d} t} \ln \mu=0$,

then the Lie symmetry will lead to the Hojman type conserved quantity

$I_{H}=\frac{1}{\mu} \frac{\partial}{\partial q_{s}}\left(\mu \xi_{s}\right)+\frac{1}{\mu} \frac{\partial}{\partial \dot{q}_{s}}\left(\mu \frac{\overline{\mathrm{d}}}{\mathrm{d} t} \xi_{s}\right)=$ const.

The complexity of the above proposition is to find the generators $\xi_{s}$ by Eq. (47) and the function $\mu$ by Eq. (49).

Example 6.1 The Lagrangian of a system is

$L=\frac{1}{2} \dot{q}^{2} \exp (-\gamma t)$,

where $\gamma$ is a constant.

Equation (22) give

$\ddot{q}=\gamma \dot{q}$.

Equation (47) give

$\frac{\overline{\mathrm{d}}}{\mathrm{d} t} \frac{\overline{\mathrm{d}}}{\mathrm{d} t} \xi=\gamma \frac{\overline{\mathrm{d}}}{\mathrm{d} t} \xi$

Equation (49) gives

$\gamma+\frac{\overline{\mathrm{d}}}{\mathrm{d} t} \ln \mu=0$.

from which one can obtain

$\xi=(\gamma q-\dot{q})^{2}, \quad \mu=\exp (-\gamma t)$.

Substitution of them in (50) gives the Hojman type conserved quantity

$I_{H}=2 \gamma(\gamma q-\dot{q})=$ const.

6.2 General holonomic system

For the system (24), the determining equations of the Lie symmetry under the transformations (46) have the form

$\frac{\overline{\mathrm{d}}}{\mathrm{d} t} \frac{\overline{\mathrm{d}}}{\mathrm{d} t} \xi_{s}=\frac{\partial \beta_{s}}{\partial q_{k}} \xi_{k}+\frac{\partial \beta_{s}}{\partial \dot{q}_{k}} \frac{\overline{\mathrm{d}}}{\mathrm{d} t} \xi_{k}$.

Proposition 6.2 For the general holonomic system (24), if the generators $\xi_{s}$ of infinitesimal transformations satisfy the determining equations (51) of the Lie symmetry and there exists a function $\mu=\mu(t, \boldsymbol{q}, \dot{\boldsymbol{q}})$ such that

$\frac{\partial \beta_{s}}{\partial \dot{q}_{s}}+\frac{\overline{\mathrm{d}}}{\mathrm{d} t} \ln \mu=0$, 
then the Lie symmetry will lead to the Hojman type conserved quantity (50).

Example 6.2 The Lagrangian and the generalized forces of a system are respectively

$L=\frac{1}{2}\left(\dot{q}_{1}^{2}+\dot{q}_{2}^{2}\right), \quad Q_{1}=0, \quad Q_{2}=\dot{q}_{1}$.

The differential equations of motion of the system have the form

$\ddot{q}_{1}=q_{1}, \quad \ddot{q}_{2}=\dot{q}_{1}$.

which are called the Whittaker equations.

Equations (51) and (52) give

$\frac{\overline{\mathrm{d}}}{\mathrm{d} t} \frac{\overline{\mathrm{d}}}{\mathrm{d} t} \xi_{1}=\xi_{1}, \quad \frac{\overline{\mathrm{d}}}{\mathrm{d} t} \frac{\overline{\mathrm{d}}}{\mathrm{d} t} \xi_{2}=\frac{\overline{\mathrm{d}}}{\mathrm{d} t} \xi_{1}, \quad 1+\frac{\overline{\mathrm{d}}}{\mathrm{d} t} \ln \mu=0$.

They have the following solutions

$\xi_{1}=0, \quad \xi_{2}=t, \quad \mu=q_{1}-\dot{q}_{2}$,

$\xi_{1}=\xi_{2}=\exp t, \quad \mu=\left(q_{1}+\dot{q}_{1}\right) \exp (-t)$,

$\xi_{1}=-\xi_{2}=\exp (-t), \quad \mu=\left(q_{1}-\dot{q}_{1}\right) \exp t$.

The corresponding Hojman type conserved quantities are respectively

$I_{H_{1}}=-\left(q_{1}-\dot{q}_{2}\right)^{-1}=C_{1}$,

$I_{H_{2}}=2\left(q_{1}+\dot{q}_{1}\right)^{-1} \exp t=C_{2}$,

$I_{H_{3}}=2\left(q_{1}-\dot{q}_{1}\right)^{-1} \exp (-t)=C_{3}$.

from which we obtain

$q_{1}=\frac{1}{C_{2}} \exp t+\frac{1}{C_{3}} \exp (-t)$,

$q_{2}=\frac{1}{C_{2}} \exp t-\frac{1}{C_{3}} \exp (-t)+\frac{1}{C_{1}} t+C_{4}$.

6.3 Nonholonomic system

Under the infinitesimal transformations (46), the determining equations of the Lie symmetry of Eq. (26) have the form

$\frac{\overline{\mathrm{d}}}{\mathrm{d} t} \frac{\overline{\mathrm{d}}}{\mathrm{d} t} \xi_{s}=\frac{\partial \gamma_{s}}{\partial q_{k}} \xi_{k}+\frac{\partial \gamma_{s}}{\partial \dot{q}_{k}} \frac{\overline{\mathrm{d}}}{\mathrm{d} t} \xi_{k}$,

Equation (28) become

$\frac{\partial f_{\beta}}{\partial q_{s}} \xi_{s}+\frac{\partial f_{\beta}}{\partial \dot{q}_{s}} \frac{\overline{\mathrm{d}}}{\mathrm{d} t} \xi_{s}=0$.

Proposition 6.3 For the nonholonomic system (10), (11), if the generators $\xi_{s}$ satisfy Eqs. (53) and (54), and there exists a function $\mu=\mu(t, \boldsymbol{q}, \dot{\boldsymbol{q}})$ such that

$\frac{\partial \gamma_{s}}{\partial \dot{q}_{s}}+\frac{\overline{\mathrm{d}}}{\mathrm{d} t} \ln \mu=0$,

then the Lie symmetry will lead to the Hojman type conserved quantity (50) [64].
Example 6.3 The Lagrangian and the constraint equation of a nonholonomic system are respectively

$L=\frac{1}{2}\left(\dot{q}_{1}^{2}+\dot{q}_{2}^{2}\right), \quad f=\dot{q}_{2}-t \dot{q}_{1}=0$.

Equation (12) give

$\ddot{q}_{1}=-\frac{t}{1+t^{2}} \dot{q}_{1}, \quad \ddot{q}_{2}=\frac{1}{1+t^{2}} \dot{q}_{1}$.

Equations (53), (54) and (55) give

$\frac{\overline{\mathrm{d}}}{\mathrm{d} t} \frac{\overline{\mathrm{d}}}{\mathrm{d} t} \xi_{1}=-\frac{t}{1+t^{2}} \frac{\overline{\mathrm{d}}}{\mathrm{d} t} \xi_{1}, \quad \frac{\overline{\mathrm{d}}}{\mathrm{d} t} \frac{\overline{\mathrm{d}}}{\mathrm{d} t} \xi_{2}=\frac{1}{1+t^{2}} \frac{\overline{\mathrm{d}}}{\mathrm{d} t} \xi_{1}$,

$\frac{\overline{\mathrm{d}}}{\mathrm{d} t} \xi_{2}-t \frac{\overline{\mathrm{d}}}{\mathrm{d} t} \xi_{1}=0$,

$-\frac{t}{1+t^{2}}+\frac{\overline{\mathrm{d}}}{\mathrm{d} t} \ln \mu=0$.

They have the following solutions

$\xi_{1}=1, \quad \xi_{2}=\left(q_{2}-\dot{q}_{1}-t \dot{q}_{2}\right)^{2}, \quad \mu=\left(1+t^{2}\right)^{1 / 2}$,

$\xi_{1}=0, \quad \xi_{2}=\dot{q}_{1}\left(1+t^{2}\right)^{1 / 2}\left(q_{2}-\dot{q}_{1}-t \dot{q}_{2}\right), \quad \mu=\left(1+t^{2}\right)^{1 / 2}$.

The corresponding Hojman type conserved quantities are respectively

$I_{H_{1}}=2\left(q_{2}-\dot{q}_{1}-t \dot{q}_{2}\right)=$ const.,

$I_{H_{2}}=\dot{q}_{1}\left(1+t^{2}\right)^{1 / 2}=$ const.

6.4 Birkhoffian system

Choose the infinitesimal transformations as

$t^{*}=t, \quad a^{\mu *}\left(t^{*}\right)=a^{\mu}(t)+\varepsilon \xi_{\mu}(t, \boldsymbol{a})$.

Under the transformations (56), the determining equations (31) of the Lie symmetry become

$\frac{\overline{\mathrm{d}}}{\mathrm{d} t} \xi_{\mu}=\frac{\partial}{\partial a^{\rho}}\left\{\Omega^{\mu \nu}\left(\frac{\partial B}{\partial a^{\nu}}+\frac{\partial R_{\nu}}{\partial t}\right)\right\} \xi_{\rho} \quad(\mu, v, \rho=1, \ldots, 2 n)$,

where

$\frac{\overline{\mathrm{d}}}{\mathrm{d} t}=\frac{\partial}{\partial t}+\Omega^{\mu \nu}\left(\frac{\partial B}{\partial a^{v}}+\frac{\partial R_{v}}{\partial t}\right) \frac{\partial}{\partial a^{\mu}}$.

Then we have

Proposition 6.4 For the Birkhoffian system (30), if the infinitesimal generators $\xi_{\mu}$ satisfy Eq. (57) and there exists a function $\mu=\mu(t, \boldsymbol{a})$ such that

$\frac{\partial}{\partial a^{\mu}}\left\{\Omega^{\mu \nu}\left(\frac{\partial B}{\partial a^{\nu}}+\frac{\partial R_{\nu}}{\partial t}\right)\right\}+\frac{\overline{\mathrm{d}}}{\mathrm{d} t} \ln \mu=0$, 
then the Lie symmetry will lead to the Hojman type conserved quantity

$I_{H}=\frac{1}{\mu} \frac{\partial}{\partial a^{v}}\left(\mu \xi_{v}\right)=$ const.

Example 6.4 The Birkhoffian and the Birkhoff's functions of a Birkhoffian system are respectively [34]

$B=\frac{1}{2}\left\{\left(a^{3}\right)^{2}+2 a^{2} a^{3}-\left(a^{4}\right)^{2}\right\}$,

$R_{1}=a^{2}+a^{3}, \quad R_{2}=0, \quad R_{3}=a^{4}, \quad R_{4}=0$.

Equations (57) and (59) give respectively

$\frac{\overline{\mathrm{d}}}{\mathrm{d} t} \xi_{1}=\xi_{3}, \quad \frac{\overline{\mathrm{d}}}{\mathrm{d} t} \xi_{2}=\xi_{4}, \quad \frac{\overline{\mathrm{d}}}{\mathrm{d} t} \xi_{3}=-\xi_{4}$,

$\frac{\overline{\mathrm{d}}}{\mathrm{d} t} \xi_{4}=-\xi_{2}, \quad \frac{\overline{\mathrm{d}}}{\mathrm{d} t} \ln \mu=0$.

They have the solutions

$\xi_{1}=\left\{a^{1}-a^{4}-\left(a^{2}+a^{3}\right) t\right\}^{2}, \quad \xi_{2}=\xi_{3}=\xi_{4}=0, \quad \mu=1$,

$\xi_{1}=t, \quad \xi_{2}=0, \quad \xi_{3}=1, \quad \xi_{4}=0, \quad \mu=a^{2}+a^{3}$.

The conserved quantity (60) gives respectively

$I_{H_{1}}=2\left\{a^{1}-a^{4}-\left(a^{2}+a^{3}\right) t\right\}=$ const.,

$I_{H_{2}}=\left(a^{2}+a^{3}\right)^{-1}=$ const.

The complexity of the study of the Hojman type conserved quantity is to find the generators $\xi_{s}$ or $\xi_{\mu}$ by using the determining equations of the Lie symmetry and the suitable function $\mu$.

\section{New conserved quantities deduced by form invariance}

For a holonomic system, a new conserved quantity can be deduced by the form invariance of the system [55]. We now present the new conserved quantity deduced by the form invariance for constrained mechanical systems, including the Lagrangian system, the general holonomic system, the nonholonomic system and the Birkhoffian system.

\subsection{Lagrangian system}

Proposition 7.1 For the Lagrangian system (1), if the generators $\xi_{0}, \xi_{s}$ of the form invariance and the gauge function $G_{F}=G_{F}(t, \boldsymbol{q}, \dot{\boldsymbol{q}})$ satisfy the structure equation

$\tilde{X}^{(1)}(L) \frac{\overline{\mathrm{d}}}{\mathrm{d} t} \xi_{0}+\tilde{X}^{(1)}\left\{\tilde{X}^{(1)}(L)\right\}+\frac{\overline{\mathrm{d}}}{\mathrm{d} t} G_{F}=0$, where

$$
\begin{aligned}
& \tilde{X}^{(1)}=\xi_{0} \frac{\partial}{\partial t}+\xi_{s} \frac{\partial}{\partial q_{s}}+\left(\frac{\overline{\mathrm{d}}}{\mathrm{d} t} \xi_{s}-\dot{q}_{s} \frac{\overline{\mathrm{d}}}{\mathrm{d} t} \xi_{0}\right) \frac{\partial}{\partial \dot{q}_{s}}, \\
& \frac{\overline{\mathrm{d}}}{\mathrm{d} t}=\frac{\partial}{\partial t}+\dot{q}_{s} \frac{\partial}{\partial q_{s}}+\alpha_{s} \frac{\partial}{\partial \dot{q}_{s}},
\end{aligned}
$$

then the form invariance will lead to the new conserved quantity

$I_{F}=\tilde{X}^{(1)}(L) \xi_{0}+\frac{\partial \tilde{X}^{(1)}(L)}{\partial \dot{q}_{s}}\left(\xi_{s}-\dot{q}_{s} \xi_{0}\right)+G_{F}=$ const.

Example 7.1 The Lagrangian of a system is

$L=t^{2}\left(\frac{1}{2} \dot{q}^{2}-\frac{1}{6} q^{6}\right)$.

We have

$X^{(1)}(L)=2 t \xi_{0}\left(\frac{1}{2} \dot{q}^{2}-\frac{1}{6} q^{6}\right)+t^{2}\left\{\dot{q}\left(\dot{\xi}-\dot{q} \dot{\xi}_{0}\right)-q^{5} \xi\right\}$.

Choosing the generators as

$\xi_{0}=t, \quad \xi=-\frac{1}{2} q$

and we have

$\tilde{X}^{(1)}(L)=X^{(1)}(L)=-L$,

$E\left\{X^{(1)}(L)\right\}=-E(L)=0$,

therefore the above generators correspond to a form invariance of the system. Substituting them into the structure equation (61), we obtain

$G_{F}=0$.

Then the conserved quantity (64) gives

$I_{F}=\frac{1}{2} t^{3} \dot{q}^{2}+\frac{1}{2} t^{2} q \dot{q}+\frac{1}{6} t^{3} q^{6}=$ const.

It must be noted that the generators $\xi_{0}, \xi$ are also the generators of a Noether symmetry, and the Noether conserved quantity deduced by them is

$I_{N}=-I_{F}$.

7.2 General holonomic system

Proposition 7.2 For the general holonomic system (8), if the generators $\xi_{0}, \xi_{\text {s }}$ of the form invariance and the gauge function $G_{F}=G_{F}(t, \boldsymbol{q}, \dot{\boldsymbol{q}})$ satisfy the structure equation

$$
\begin{aligned}
& \tilde{X}^{(1)}(L) \frac{\overline{\mathrm{d}}}{\mathrm{d} t} \xi_{0}+\tilde{X}^{(1)}\left\{\tilde{X}^{(1)}(L)\right\}+\tilde{X}^{(1)}\left(Q_{s}\right)\left(\xi_{s}-\dot{q}_{s} \xi_{0}\right) \\
& +\frac{\overline{\mathrm{d}}}{\mathrm{d} t} G_{F}=0
\end{aligned}
$$


where

$\frac{\overline{\mathrm{d}}}{\mathrm{d} t}=\frac{\partial}{\partial t}+\dot{q}_{s} \frac{\partial}{\partial q_{s}}+\beta_{s} \frac{\partial}{\partial \dot{q}_{s}}$,

then the form invariance will lead to the new conserved quantity (64).

Obviously, Proposition 7.1 is a particular case of Proposition 7.2.

Example 7.2 A system with two-degree-of-freedom is

$L=\frac{1}{2}\left(\dot{q}_{1}^{2}+\dot{q}_{2}^{2}\right)-q_{2}$,

$Q_{1}=\frac{1}{1+t^{2}}\left(\dot{q}_{2}-2 t \dot{q}_{1}-t\right)$,

$Q_{2}=-\frac{1}{1+t^{2}}\left(t \dot{q}_{2}+2 \dot{q}_{1}-t^{2}\right)$.

We can obtain the following result

$\xi_{0}=\xi_{1}=0, \quad \xi_{2}=t \dot{q}_{1}+\dot{q}_{2}+q_{1}+t, \quad G_{F}=0$,

$I_{F}=-\left(t \dot{q}_{1}+\dot{q}_{2}+q_{1}+t\right)=$ const.

7.3 Nonholonomic system

Proposition 7.3 For the nonholonomic system (10), (11), if the generators $\xi_{0}, \xi_{s}$ of the form invariance and the gauge function $G_{F}=G_{F}(t, \boldsymbol{q}, \dot{\boldsymbol{q}})$ satisfy the structure equation

$$
\begin{aligned}
& \tilde{X}^{(1)}(L) \frac{\overline{\mathrm{d}}}{\mathrm{d} t} \xi_{0}+\tilde{X}^{(1)}\left\{\tilde{X}^{(1)}(L)\right\}+\tilde{X}^{(1)}\left(Q_{s}+\Lambda_{s}\right)\left(\xi_{s}-\dot{q}_{s} \xi_{0}\right) \\
& +\frac{\overline{\mathrm{d}}}{\mathrm{d} t} G_{F}=0
\end{aligned}
$$

where

$\frac{\overline{\mathrm{d}}}{\mathrm{d} t}=\frac{\partial}{\partial t}+\dot{q}_{s} \frac{\partial}{\partial q_{s}}+\gamma_{s} \frac{\partial}{\partial \dot{q}_{s}}$,

then the form invariance will lead to the conserved quantity (64).

Example 7.3 Novoselov problem is [25]

$L=\frac{1}{2}\left(\dot{q}_{1}^{2}+\dot{q}_{2}^{2}\right), \quad f=\dot{q}_{1}+b t \dot{q}_{2}-b q_{2}+t=0$.

By Proposition 7.3, we can obtain the result

$\xi_{0}=0, \quad \xi_{1}=\dot{q}_{2}-b t \dot{q}_{1}+b q_{1}+b t, \quad \xi_{2}=1, \quad G_{F}=-b^{2} t$,

$I_{F}=b\left(\dot{q}_{2}-b t \dot{q}_{1}+b q_{1}\right)=$ const.

\subsection{Birkhoffian system}

Proposition 7.4 For the Birkhoffian system (17), if the generators $\xi_{0}, \xi_{\mu}$ of the form invariance and the gauge function $G_{F}=G_{F}(t, a)$ satisfy the structure equation

$$
\begin{aligned}
& X^{(0)}\left\{X^{(0)}\left(R_{\mu}\right)\right\} \Omega^{\mu \nu}\left(\frac{\partial B}{\partial a^{v}}+\frac{\partial R_{v}}{\partial t}\right)-X^{(0)}\left\{X^{(0)}(B)\right\} \\
& -X^{(0)}(B) \frac{\overline{\mathrm{d}}}{\mathrm{d} t} \xi_{0}+X^{(0)}\left(R_{\mu}\right) \frac{\overline{\mathrm{d}}}{\mathrm{d} t} \xi_{\mu}+\frac{\overline{\mathrm{d}}}{\mathrm{d} t} G_{F}=0
\end{aligned}
$$

where

$\frac{\overline{\mathrm{d}}}{\mathrm{d} t}=\frac{\partial}{\partial t}+\Omega^{\mu \nu}\left(\frac{\partial B}{\partial a^{\nu}}+\frac{\partial R_{v}}{\partial t}\right) \frac{\partial}{\partial a^{\mu}}$,

then the form invariance will lead to the conserved quantity

$I_{F}=X^{(0)}\left(R_{\mu}\right) \xi_{\mu}-X^{(0)}(B) \xi_{0}+G_{F}=$ const.

Example 7.4 A Birkhoffian system of four order is

$$
\begin{aligned}
B= & \left(a^{2} \sin t+a^{4} \cos t\right)^{2}, \\
R_{1}= & 0, \quad R_{2}=a^{1}-a^{4}-\left(a^{2}+a^{3}\right) t \\
& +\left(a^{2} \sin t+a^{4} \cos t\right) \cos t, \\
R_{3}= & a^{1}-a^{4}-\left(a^{2}+a^{3}\right) t, \\
R_{4}= & -\left(a^{2} \sin t+a^{4} \cos t\right) \sin t .
\end{aligned}
$$

We can obtain the result

$\xi_{0}=0, \quad \xi_{1}=\left(a^{2}+a^{3}\right) t+1, \quad \xi_{3}=a^{2}+a^{3}$,

$\xi_{2}=\xi_{4}=0, \quad G_{F}=0, \quad I_{F}=a^{2}+a^{3}=$ const.

The complexity of the study on the new conserved quantity for the constrained mechanical systems is to find the generators $\xi_{0}, \xi_{s}\left(\right.$ or $\left.\xi_{\mu}\right)$ of the form invariance and the suitable gauge function $G_{F}$.

\section{Non-Noether conserved quantities deduced by Noether symmetry}

In Sect. 5, we have presented the Noether conserved quantity deduced by the Noether symmetry for the constrained mechanical systems. In fact, we can also find the non-Noether conserved quantity, say the Hojman type conserved quantity and the new conserved quantity, by using the Noether symmetry for the constrained mechanical systems.

\subsection{Lagrangian system}

Under the infinitesimal transformations (46), the Noether identity (6) of the Lagrangian system (1) becomes

$$
\frac{\partial L}{\partial q_{s}} \xi_{s}+\frac{\partial L}{\partial \dot{q}_{s}} \dot{\xi}_{s}+\dot{G}_{N}=0 .
$$

Proposition 8.1 For the Lagrangian system (1), if the generators $\xi_{s}$ of the Noether symmetry satisfy Eq. (47) and there exists a function $\mu=\mu(t, \boldsymbol{q}, \dot{\boldsymbol{q}})$ satisfying $E q$. (49), then the Noether symmetry will lead to the Hojman type conserved quantity (50).

Example 8.1 The Lagrangian of a system is

$L=\frac{1}{2}\left(\dot{q}_{1}^{2}+\dot{q}_{2}^{2}\right)-q_{2}$. 
Identity (72), Eqs. (47) and (49) give respectively

$\dot{q}_{1} \dot{\xi}_{1}+\dot{q}_{2} \dot{\xi}_{2}-\xi_{2}+\dot{G}_{N}=0$,

$\frac{\overline{\mathrm{d}}}{\mathrm{d} t} \frac{\overline{\mathrm{d}}}{\mathrm{d} t} \xi_{1}=0, \quad \frac{\overline{\mathrm{d}}}{\mathrm{d} t} \frac{\overline{\mathrm{d}}}{\mathrm{d} t} \xi_{2}=0$,

$\frac{\overline{\mathrm{d}}}{\mathrm{d} t} \ln \mu=0$.

which have the solutions

$\xi_{1}=1, \quad \xi_{2}=0, \quad G_{N}=0, \quad \mu=q_{1}-\dot{q}_{1} t$,

$\xi_{1}=0, \quad \xi_{2}=1, \quad G_{N}=t, \quad \mu=q_{2}-\dot{q}_{2} t-\frac{1}{2} t^{2}$.

The conserved quantity (50) gives respectively

$I_{H_{1}}=\left(q_{1}-\dot{q}_{1} t\right)^{-1}=$ const.,

$I_{H_{2}}=\left(q_{2}-\dot{q}_{2} t-\frac{1}{2} t^{2}\right)^{-1}=$ const.,

and the Noether conserved quantity are respectively

$I_{N_{1}}=\dot{q}_{1}=$ const.,

$I_{N_{2}}=\dot{q}_{2}+t=$ const.

Proposition 8.2 For the Lagrangian system (1), if the generators $\xi_{0}, \xi_{s}$ of the Noether symmetry satisfy Eq. (34) and there exists a gauge function $G_{F}=G_{F}(t, \boldsymbol{q}, \dot{\boldsymbol{q}})$ satisfying $E q$. (61), then the Noether symmetry will lead to the new conserved quantity (64).

\subsection{General holonomic system}

Under the infinitesimal transformations (46), the Noether identity (9) of the general holonomic system becomes

$\frac{\partial L}{\partial q_{s}} \xi_{s}+\frac{\partial L}{\partial \dot{q}_{s}} \dot{\xi}_{s}+Q_{s} \xi_{s}+\dot{G}_{N}=0$.

Proposition 8.3 For the general holonomic system (8), if the generators $\xi_{s}$ of the Noether symmetry satisfy Eq. (51) and there exists a function $\mu=\mu(t, \boldsymbol{q}, \dot{\boldsymbol{q}})$ satisfying $E q$. (52), then the Noether symmetry will lead to the Hojman type conserved quantity (50).

Proposition 8.4 For the general holonomic system (8), if the generators $\xi_{0}, \xi_{s}$ of the Noether symmetry satisfy Eq. (36) and there exists a gauge function $G_{F}=G_{F}(t, \boldsymbol{q}, \dot{\boldsymbol{q}})$ satisfying Eq. (65), then the Noether symmetry will lead to the new conserved quantity (64).

Example 8.2 The Lagrangian and the generalized forces of a holonomic system are respectively

$L=\frac{1}{2}\left(\dot{q}_{1}^{2}+\dot{q}_{2}^{2}+\dot{q}_{3}^{2}\right)-q_{3}$,

$Q_{1}=-\dot{q}_{2}^{2}, \quad Q_{2}=\dot{q}_{1}^{2}, \quad Q_{3}=0$.
The Noether identity (9) gives

$$
\begin{aligned}
& L \dot{\xi}_{0}+\dot{q}_{1}\left(\dot{\xi}_{1}-\dot{q}_{1} \dot{\xi}_{0}\right)+\dot{q}_{2}\left(\dot{\xi}_{2}-\dot{q}_{2} \dot{\xi}_{0}\right)+\dot{q}_{3}\left(\dot{\xi}_{3}-\dot{q}_{3} \dot{\xi}_{0}\right)-\xi_{3} \\
& \quad-\dot{q}_{2}^{2}\left(\xi_{1}-\dot{q}_{1} \xi_{0}\right)+\dot{q}_{1}^{2}\left(\xi_{2}-\dot{q}_{2} \xi_{0}\right)+\dot{G}_{N}=0 .
\end{aligned}
$$

It has the solution

$\xi_{0}=\xi_{1}=\xi_{2}=0, \quad \xi_{3}=-\dot{q}_{3}-t, \quad G_{N}=\frac{1}{2} \dot{q}_{3}^{2}-\frac{1}{2} t^{2}$.

We have

$\tilde{X}^{(1)}(L)=\dot{q}_{3}+t$,

$E_{S}\left\{\tilde{X}^{(1)}(L)\right\}=\tilde{X}^{(1)}\left(Q_{s}\right)=0 \quad(s=1,2,3)$,

$G_{F}=0$,

and the conserved quantity (64) gives

$I_{F}=-\dot{q}_{3}-t=$ const.

It must be noted that the Noether conserved quantity deduced by the Noether symmetry is

$I_{N}=-\frac{1}{2} \dot{q}_{3}^{2}-\dot{q}_{3} t-\frac{1}{2} t^{2}=$ const.

Therefore, the conserved quantity $I_{F}$ is a non-Noether one.

\subsection{Nonholonomic system}

Under the infinitesimal transformations (46), the Noether identity (14) of the nonholonomic system becomes

$$
\frac{\partial L}{\partial q_{s}} \xi_{s}+\frac{\partial L}{\partial \dot{q}_{s}} \dot{\xi}_{s}+\left(Q_{s}+\Lambda_{s}\right) \xi_{s}+\dot{G}_{N}=0,
$$

and Eq. (15) become

$$
\frac{\partial f_{\beta}}{\partial \dot{q}_{s}} \xi_{s}=0 .
$$

Proposition 8.5 For the nonholonomic system (10), (11), if the generators $\xi_{s}$ of the Noether symmetry satisfy Eqs. (53), (54) and there exists a function $\mu=\mu(t, \boldsymbol{q}, \dot{\boldsymbol{q}})$ satisfying $E q$. (55), then the Noether symmetry will lead to the Hojman type conserved quantity (50).

Proposition 8.6 For the nonholonomic system (10), (11), if the generators $\xi_{0}, \xi_{s}$ of the Noether symmetry satisfy Eqs. (38), (40) and there exists a gauge function $G_{F}=$ $G_{F}(t, \boldsymbol{q}, \dot{\boldsymbol{q}})$ satisfying $E q$. (67), then the Noether symmetry will lead to the new conserved quantity (64).

Example 8.3 Rolling sphere problem.

The Lagrangian and the constraint equations of the problem are respectively

$$
\begin{aligned}
& L=\frac{1}{2} m\left(\dot{x}^{2}+\dot{y}^{2}\right)+\frac{1}{2} \frac{2}{5} m a^{2}\left(\dot{\psi}^{2}+\dot{\theta}^{2}+\dot{\varphi}^{2}+2 \dot{\psi} \dot{\varphi} \cos \theta\right), \\
& f_{1}=\dot{x}+a(\dot{\varphi} \sin \theta \cos \psi-\dot{\theta} \sin \psi)=0, \\
& f_{2}=\dot{y}+a(\dot{\varphi} \sin \theta \sin \psi+\dot{\theta} \cos \psi)=0,
\end{aligned}
$$


where $a$ is the radius of the sphere, $m$ the mass, $x$ and $y$ the coordinates of the center of the sphere, $\psi, \theta$ and $\varphi$ the Euler angles.

Let

$q_{1}=\psi, \quad q_{2}=\theta, \quad q_{3}=\varphi, \quad q_{4}=x, \quad q_{5}=y$.

Then Eq. (11) give

$$
\begin{aligned}
& \frac{2}{5} m a^{2}\left(\dot{q}_{1}+\dot{q}_{3} \cos q_{2}\right)=0, \\
& \frac{2}{5} m a^{2} \ddot{q}_{2}+\frac{2}{5} m a^{2} \dot{q}_{1} \dot{q}_{3} \sin q_{2}=-\lambda_{1} a \sin q_{1}+\lambda_{2} a \cos q_{1}, \\
& \frac{2}{5} m a^{2}\left(\dot{q}_{3}+\dot{q}_{1} \cos q_{2}\right)=\lambda_{1} a \sin q_{2} \cos q_{1} \\
& \quad+\lambda_{2} a \sin q_{2} \sin q_{1}, \\
& m \ddot{q}_{4}=\lambda_{1}, \\
& m \ddot{q}_{5}=\lambda_{2} .
\end{aligned}
$$

By using the constraint equations, we obtain [32]

$\lambda_{1}=\lambda_{2}=0$,

and then

$Q_{s}+\Lambda_{s}=0 \quad(s=1,2,3,4,5)$.

All of the accelerations $\ddot{q}_{s}$ can be solved as

$\ddot{q}_{1}=\frac{1}{\sin q_{2}} \dot{q}_{2} \dot{q}_{3}-\frac{\cos q_{2}}{\sin q_{2}} \dot{q}_{1} \dot{q}_{2}$,

$\ddot{q}_{2}=-\dot{q}_{1} \dot{q}_{3} \sin q_{2}$,

$\ddot{q}_{3}=\frac{1}{\sin q_{2}} \dot{q}_{1} \dot{q}_{2}-\frac{\cos q_{2}}{\sin q_{2}} \dot{q}_{2} \dot{q}_{3}$,

$\ddot{q}_{4}=0$,

$\ddot{q}_{5}=0$.

Equation (53) give

$$
\begin{aligned}
\frac{\overline{\mathrm{d}}}{\mathrm{d} t} \frac{\overline{\mathrm{d}}}{\mathrm{d} t} \xi_{1}= & \frac{1}{\sin q_{2}}\left(\dot{q}_{3} \frac{\overline{\mathrm{d}}}{\mathrm{d} t} \xi_{2}+\dot{q}_{2} \frac{\overline{\mathrm{d}}}{\mathrm{d} t} \xi_{3}\right)-\frac{\cos q_{2}}{\sin ^{2} q_{2}} \dot{q}_{2} \dot{q}_{3} \xi_{2} \\
& -\frac{\cos q_{2}}{\sin q_{2}}\left(\dot{q}_{1} \frac{\overline{\mathrm{d}}}{\mathrm{d} t} \xi_{2}+\dot{q}_{2} \frac{\overline{\mathrm{d}}}{\mathrm{d} t} \xi_{1}\right)+\frac{1}{\sin ^{2} q_{2}} \dot{q}_{1} \dot{q}_{2} \xi_{2},
\end{aligned}
$$

$\frac{\overline{\mathrm{d}}}{\mathrm{d} t} \frac{\overline{\mathrm{d}}}{\mathrm{d} t} \xi_{2}=-\dot{q}_{1} \frac{\overline{\mathrm{d}}}{\mathrm{d} t} \xi_{3} \sin q_{2}-\dot{q}_{3} \frac{\overline{\mathrm{d}}}{\mathrm{d} t} \xi_{1} \sin q_{2}-\dot{q}_{1} \dot{q}_{3} \xi_{2} \cos q_{2}$,

$$
\begin{aligned}
\frac{\overline{\mathrm{d}}}{\mathrm{d} t} \frac{\overline{\mathrm{d}}}{\mathrm{d} t} \xi_{3}= & \frac{1}{\sin q_{2}}\left(\dot{q}_{1} \frac{\overline{\mathrm{d}}}{\mathrm{d} t} \xi_{2}+\dot{q}_{2} \frac{\overline{\mathrm{d}}}{\mathrm{d} t} \xi_{1}\right)-\frac{\cos q_{2}}{\sin ^{2} q_{2}} \dot{q}_{1} \dot{q}_{2} \xi_{2} \\
& -\frac{\cos q_{2}}{\sin q_{2}}\left(\dot{q}_{2} \frac{\overline{\mathrm{d}}}{\mathrm{d} t} \xi_{3}+\dot{q}_{3} \frac{\overline{\mathrm{d}}}{\mathrm{d} t} \xi_{2}\right)+\frac{1}{\sin ^{2} q_{2}} \dot{q}_{2} \dot{q}_{3} \xi_{2},
\end{aligned}
$$

$$
\begin{gathered}
\frac{\overline{\mathrm{d}}}{\mathrm{d} t} \frac{\overline{\mathrm{d}}}{\mathrm{d} t} \xi_{4}=0, \\
\frac{\overline{\mathrm{d}}}{\mathrm{d} t} \frac{\overline{\mathrm{d}}}{\mathrm{d} t} \xi_{5}=0 .
\end{gathered}
$$

Equation (54) show

$\frac{\overline{\mathrm{d}}}{\mathrm{d} t} \xi_{4}+a\left[\frac{\overline{\mathrm{d}}}{\mathrm{d} t} \xi_{3} \sin q_{2} \cos q_{1}-\frac{\overline{\mathrm{d}}}{\mathrm{d} t} \xi_{2} \sin q_{1}\right]$

$+a\left[\dot{q}_{3} \xi_{2} \cos q_{2} \cos q_{1}-\dot{q}_{3} \xi_{1} \sin q_{2} \sin q_{1}-\dot{q}_{2} \xi_{1} \cos q_{1}\right]=0$,

$\frac{\overline{\mathrm{d}}}{\mathrm{d} t} \xi_{5}+a\left[\frac{\overline{\mathrm{d}}}{\mathrm{d} t} \xi_{3} \sin q_{2} \sin q_{1}+\frac{\overline{\mathrm{d}}}{\mathrm{d} t} \xi_{2} \cos q_{1}\right]$

$+a\left[\dot{q}_{3} \xi_{2} \cos q_{2} \sin q_{1}+\dot{q}_{3} \xi_{1} \sin q_{2} \cos q_{1}-\dot{q}_{2} \xi_{1} \sin q_{1}\right]=0$.

Identity (74) becomes

$\begin{aligned}- & \frac{2}{5} m a^{2} \dot{q}_{1} \dot{q}_{3} \xi_{2} \sin q_{2}+\frac{2}{5} m a^{2} \dot{q}_{1} \dot{\xi}_{1}+\frac{2}{5} m a^{2} \dot{q}_{2} \dot{\xi}_{2}+\frac{2}{5} m a^{2} \dot{q}_{3} \dot{\xi}_{3} \\ & +\frac{2}{5} m a^{2}\left(\dot{q}_{1} \dot{\xi}_{3}+\dot{q}_{3} \dot{\xi}_{1}\right) \cos q_{2}+m \dot{q}_{4} \dot{\xi}_{4}+m \dot{q}_{5} \dot{\xi}_{5}+\dot{G}_{N}=0 .\end{aligned}$

Eq. (55) gives

$\frac{\overline{\mathrm{d}}}{\mathrm{d} t} \ln \mu=0$.

The above 9 equations have the solutions

$\xi_{4}=1, \quad \xi_{1}=\xi_{2}=\xi_{3}=\xi_{5}=0, \quad \mu=q_{4}-\dot{q}_{4} t$,

$\xi_{5}=1, \quad \xi_{1}=\xi_{2}=\xi_{3}=\xi_{4}=0, \quad \mu=q_{5}-\dot{q}_{5} t$.

The Hojman type conserved quantity are respectively

$I_{H_{1}}=\left(q_{4}-\dot{q}_{4} t\right)^{-1}=$ const.

$I_{H_{2}}=\left(q_{5}-\dot{q}_{5} t\right)^{-1}=$ const.

\subsection{Birkhoffian system}

Under the infinitesimal transformations (56), the Noether identity (20) becomes

$$
\left(\frac{\partial R_{v}}{\partial a^{\mu}} \dot{a}^{\nu}-\frac{\partial B}{\partial a^{\mu}}\right) \xi_{\mu}+R_{\mu} \dot{\xi}_{\mu}+\dot{G}_{N}=0 .
$$

Proposition 8.7 For the Birkhoffian system (17), if the generators $\xi_{\mu}$ of the Noether symmetry satisfy Eq. (57) and there exists a function $\mu=\mu(t, \boldsymbol{a})$ satisfying Eq. (59), then the Noether symmetry will lead to the Hojman type conserved quantity (60).

Proposition 8.8 For the Birkhoffian system (17), if the generators $\xi_{0}, \xi_{\mu}$ of the Noether symmetry satisfy Eq. (42) and the generators and the gauge function $G_{F}=G_{F}(t, a)$ satisfy Eq. (69), then the Noether symmetry will lead to the new conserved quantity (71).

Example 8.4 A four order Birkhoffian system is

$$
\begin{aligned}
& B=a^{2}+\frac{1}{2}\left\{\left(a^{3}\right)^{2}+\left(a^{4}\right)^{2}\right\}, \\
& R_{1}=a^{3}, \quad R_{2}=a^{4}, \quad R_{3}=R_{4}=0 .
\end{aligned}
$$


The Noether identity (20) gives

$$
\begin{aligned}
& \left(\dot{a}^{1}-a^{3}\right) \xi_{3}+\left(\dot{a}^{2}-a^{4}\right) \xi_{4}-\xi_{2} \\
& \quad-\left\{a^{2}+\frac{1}{2}\left[\left(a^{3}\right)^{2}+\left(a^{4}\right)^{2}\right]\right\} \dot{\xi}_{0}+a^{3} \dot{\xi}_{1} \\
& +a^{4} \dot{\xi}_{2}+\dot{G}_{N}=0 .
\end{aligned}
$$

Equation (42) give

$$
\begin{aligned}
& \left(\frac{\partial \xi_{4}}{\partial a^{1}}-\frac{\partial \xi_{3}}{\partial a^{2}}\right) \dot{a}^{2}-\frac{\partial \xi_{3}}{\partial a^{3}} \dot{a}^{3}-\frac{\partial \xi_{3}}{\partial a^{4}} \dot{a}^{4} \\
& -\left(\frac{\partial \xi_{2}}{\partial a^{1}}+a^{3} \frac{\partial \xi_{3}}{\partial a^{1}}+a^{4} \frac{\partial \xi_{4}}{\partial a^{1}}\right)=0 \\
& \left(\frac{\partial \xi_{3}}{\partial a^{2}}-\frac{\partial \xi_{4}}{\partial a^{1}}\right) \dot{a}^{1}-\frac{\partial \xi_{4}}{\partial a^{3}} \dot{a}^{3} \\
& -\frac{\partial \xi_{4}}{\partial a^{4}} \dot{a}^{4}-\left(\frac{\partial \xi_{2}}{\partial a^{2}}+a^{3} \frac{\partial \xi_{3}}{\partial a^{2}}+a^{4} \frac{\partial \xi_{4}}{\partial a^{2}}\right)=0 \\
& \frac{\partial \xi_{3}}{\partial a^{3}} \dot{a}^{1}+\frac{\partial \xi_{4}}{\partial a^{3}} \dot{a}^{2}-\left(\frac{\partial \xi_{2}}{\partial a^{3}}+\xi_{3}+a^{3} \frac{\partial \xi_{3}}{\partial a^{3}}+a^{4} \frac{\partial \xi_{4}}{\partial a^{3}}\right)=0 \\
& \frac{\partial \xi_{3}}{\partial a^{4}} \dot{a}^{1}+\frac{\partial \xi_{4}}{\partial a^{4}} \dot{a}^{2}-\left(\frac{\partial \xi_{2}}{\partial a^{4}}+\xi_{4}+a^{3} \frac{\partial \xi_{3}}{\partial a^{4}}+\frac{\partial \xi_{4}}{\partial a^{4}}\right)=0
\end{aligned}
$$

Eq. (69) becomes

$$
\begin{aligned}
& \left(\frac{\partial \xi_{3}}{\partial a^{\mu}} \xi_{\mu}+\frac{\partial \xi_{3}}{\partial t} \xi_{0}\right) a^{3}+\left(\frac{\partial \xi_{4}}{\partial a^{\mu}} \xi_{\mu}+\frac{\partial \xi_{4}}{\partial t} \xi_{0}\right) a^{4} \\
& -X^{(0)}\left(\xi_{2}+a^{3} \xi_{3}+a^{4} \xi_{4}\right) \\
& -\left(\xi_{2}+a^{3} \xi_{3}+a^{4} \xi_{4}\right) \frac{\overline{\mathrm{d}}}{\mathrm{d} t} \xi_{0}+\xi_{3} \frac{\overline{\mathrm{d}}}{\mathrm{d} t} \xi_{1}+\xi_{4} \frac{\overline{\mathrm{d}}}{\mathrm{d} t} \xi_{2} \\
& +\frac{\overline{\mathrm{d}}}{\mathrm{d} t} G_{F}=0 .
\end{aligned}
$$

These 6 equations have the following solution

$\xi_{0}=\xi_{1}=\xi_{3}=0, \quad \xi_{2}=a^{4}, \quad \xi_{4}=-1, \quad G_{F}=-t$.

The conserved quantity (71) gives

$I_{F}=-a^{4}-t=$ const.

This is a new conserved quantity deduced by the Noether symmetry. It must be noted that the Noether conserved quantity deduced by the Noether symmetry is

$I_{N}=\frac{1}{2}\left(a^{4}\right)^{2}+a^{2}=$ const.

therefore, the conserved quantity $I_{F}$ is a non-Noether one.

\section{Conserved quantities deduced by Lie symmetry}

In Sect. 6, we have presented the Hojman type conserved quantities deduced by the Lie symmetry for the constrained mechanical systems. In fact, the Noether conserved quantity and the new conserved quantity can also be deduced by using the Lie symmetry for the systems.

\subsection{Lagrangian system}

Proposition 9.1 For the Lagrangian system (1), if the generators $\xi_{0}, \xi_{s}$ of the Lie symmetry and the gauge function $G_{N}=G_{N}(t, \boldsymbol{q}, \dot{\boldsymbol{q}})$ satisfy the Noether identity (6), then the Lie symmetry will lead to the Noether conserved quantity (43).

Proposition 9.2 For the Lagrangian system (1), if the generators $\xi_{0}, \xi_{s}$ of the Lie symmetry satisfy Eq. (34) and the generators and the gauge function $G_{F}=G_{F}(t, \boldsymbol{q}, \dot{\boldsymbol{q}})$ satisfy Eq. (61), then the Lie symmetry will lead to the new conserved quantity (64).

Example 9.1 The Lagrangian of a system is

$L=\frac{1}{2}\left(\dot{q}_{1}^{2}+\dot{q}_{2}^{2}\right)-\left(q_{1}^{2}+q_{2}^{2}+q_{1}^{4}+q_{2}^{4}+6 q_{1}^{2} q_{2}^{2}\right)$

Equation (23) give

$$
\begin{aligned}
& \ddot{\xi}_{1}-\dot{q}_{1} \ddot{\xi}_{0}+2 \dot{\xi}_{0}\left(2 q_{1}+4 q_{1}^{3}+12 q_{1} q_{2}^{2}\right)=-2 \xi_{1} \\
& \quad-12 q_{1}^{2} \xi_{1}-12 \xi_{1} q_{2}^{2}-24 q_{1} q_{2} \xi_{2}, \\
& \ddot{\xi}_{2}-\dot{q}_{2} \ddot{\xi}_{0}+2 \dot{\xi}_{0}\left(2 q_{2}+4 q_{2}^{3}+12 q_{2} q_{1}^{2}\right)=-2 \xi_{2} \\
& \quad-12 q_{2}^{2} \xi_{2}-12 \xi_{2} q_{1}^{2}-24 q_{1} q_{2} \xi_{1} .
\end{aligned}
$$

The Noether identity (6) gives

$$
\begin{gathered}
L \dot{\xi}_{0}+\dot{q}_{1}\left(\dot{\xi}_{1}-\dot{q}_{1} \dot{\xi}_{0}\right)+\dot{q}_{2}\left(\dot{\xi}_{2}-\dot{q}_{2} \dot{\xi}_{0}\right)-2 q_{1} \xi_{1}-2 q_{2} \xi_{2} \\
-4 q_{1}^{3} \xi_{1}-4 q_{2}^{3} \xi_{2}-12 q_{1} q_{2}^{2} \xi_{1}-12 q_{1}^{2} q_{2} \xi_{2}+\dot{G}_{N}=0 .
\end{gathered}
$$

These three equations have the solution

$$
\begin{aligned}
& \xi_{0}=0, \quad \xi_{1}=\dot{q}_{2}, \quad \xi_{2}=\dot{q}_{1}, \\
& \quad G_{N}=-\dot{q}_{1} \dot{q}_{2}+2 q_{1} q_{2}+4 q_{1} q_{2}\left(q_{1}^{2}+q_{2}^{2}\right) .
\end{aligned}
$$

The Noether conserved quantity (43) gives

$$
I_{N}=\dot{q}_{1} \dot{q}_{2}+2 q_{1} q_{2}+4 q_{1} q_{2}\left(q_{1}^{2}+q_{2}^{2}\right)=\text { const. }
$$

\subsection{General holonomic system}

For the general holonomic system, the Lie symmetry can lead to the Noether conserved quantity and the new conserved quantity. We have

Proposition 9.3 For the general holonomic system (8), if the generators $\xi_{0}, \xi_{s}$ of the Lie symmetry and the gauge function $G_{N}=G_{N}(t, \boldsymbol{q}, \dot{\boldsymbol{q}})$ satisfy the Noether identity (9), then the Lie symmetry will lead to the Noether conserved quantity (43). 
Proposition 9.4 For the general holonomic system (8), if the generators $\xi_{0}, \xi_{\text {s }}$ of the Lie symmetry satisfy Eq. (36) and the generators and the gauge function $G_{F}=G_{F}(t, \boldsymbol{q}, \dot{\boldsymbol{q}})$ satisfy $E q .(65)$, then the Lie symmetry will lead to the new conserved quantity (64).

Example 9.2 The Lagrangian and the generalized forces of a system with three-degree-of-freedom are respectively

$L=\frac{1}{2}\left(\dot{q}_{1}^{2}+\dot{q}_{2}^{2}+\dot{q}_{3}^{2}\right)-q_{3}$,

$Q_{1}=-\dot{q}_{2}^{3}, \quad Q_{2}=\dot{q}_{1}^{3}, \quad Q_{3}=0$.

Equation (25) give

$$
\begin{aligned}
\ddot{\xi}_{1}-\dot{q}_{1} \ddot{\xi}_{0}-2 \dot{\xi}_{0}\left(-\dot{q}_{2}^{3}\right) & =-3 \dot{q}_{2}^{2}\left(\dot{\xi}_{2}-\dot{q}_{2} \dot{\xi}_{0}\right), \\
\ddot{\xi}_{2}-\dot{q}_{2} \ddot{\xi}_{0}-2 \dot{\xi}_{0}\left(-\dot{q}_{1}^{3}\right) & =3 \dot{q}_{1}^{2}\left(\dot{\xi}_{1}-\dot{q}_{1} \dot{\xi}_{0}\right), \\
\ddot{\xi}_{3}-\dot{q}_{3} \ddot{\xi}_{0}-2 \dot{\xi}_{0}(-1) & =0 .
\end{aligned}
$$

They have the solution

$\xi_{0}=\xi_{1}=\xi_{2}=0, \quad \xi_{3}=\dot{q}_{3}+t$,

and then we have

$\tilde{X}^{(1)}(L)=-\dot{q}_{3}-t, \quad E_{S}\left\{\tilde{X}^{(1)}(L)\right\}=0$,

$\tilde{X}^{(1)}\left(Q_{s}\right)=0 \quad(s=1,2,3)$.

Equation (65) gives

$G_{F}=0$.

Thus, the conserved quantity (64) gives

$I_{F}=-\dot{q}_{3}-t=$ const.

The conserved quantity is a non-Noether one, because the Noether conserved quantity deduced by the above generators is

$I_{N}=\frac{1}{2} \dot{q}_{3}^{2}+t \dot{q}_{3}+\frac{1}{2} t^{2}=$ const.

9.3 Nonholonomic system

For the nonholonomic system, the Lie symmetry can lead to the Noether conserved quantity and the new conserved quantity. We have

Proposition 9.5 For the nonholonomic system (10), (11), if the generators $\xi_{0}, \xi_{s}$ of the Lie symmetry and the gauge function $G_{N}=G_{N}(t, \boldsymbol{q}, \dot{\boldsymbol{q}})$ satisfy the Noether identity (14) and Eq. (15), then the Lie symmetry will lead to the Noether conserved quantity (43).

Proposition 9.6 For the nonholonomic system (10), (11), if the generators $\xi_{0}, \xi_{s}$ of the Lie symmetry satisfy Eqs. (38) and (40), and the generators and the gauge function $G_{F}=$ $G_{F}(t, \boldsymbol{q}, \dot{\boldsymbol{q}})$ satisfy Eq. (67), then the Lie symmetry will lead to the new conserved quantity (64).
Example 9.3 Chaplygin sled problem.

The Lagrangian and the nonholonomic constraint equation are respectively [32]

$L=\frac{1}{2} m\left(\dot{q}_{1}^{2}+\dot{q}_{2}^{2}\right)+\frac{1}{2} J \dot{q}_{3}^{2}-\frac{1}{2} k q_{3}^{2}$,

$f=\dot{q}_{2}-\dot{q}_{1} \tan q_{3}=0$.

Equation (27) give

$\ddot{\xi}_{1}-\dot{q}_{1} \ddot{\xi}_{0}-2 \dot{\xi}_{0}\left(-\dot{q}_{1} \dot{q}_{3} \tan q_{3}\right)=-\frac{\dot{q}_{1} \dot{q}_{3}}{\cos ^{2} q_{3}} \xi_{3}$

$+\left(\dot{\xi}_{1}-\dot{q}_{1} \dot{\xi}_{0}\right)\left(-\dot{q}_{3} \tan q_{3}\right)+\left(\dot{\xi}_{3}-\dot{q}_{3} \dot{\xi}_{0}\right)\left(-\dot{q}_{1} \tan q_{3}\right)$,

$\ddot{\xi}_{2}-\dot{q}_{2} \ddot{\xi}_{0}-2 \dot{\xi}_{0}\left(\dot{q}_{1} \dot{q}_{3}\right)=\dot{q}_{3}\left(\dot{\xi}_{1}-\dot{q}_{1} \dot{\xi}_{0}\right)+\dot{q}_{1}\left(\dot{\xi}_{3}-\dot{q}_{3} \dot{\xi}_{0}\right)$,

$\ddot{\xi}_{3}-\dot{q}_{3} \ddot{\xi}_{0}-2 \dot{\xi}_{0}\left(-\frac{k}{J} q_{3}\right)=-\frac{k}{J} \xi_{3}$.

Equation (28) shows

$\dot{\xi}_{2}-\dot{q}_{2} \dot{\xi}_{0}-\left(\dot{\xi}_{1}-\dot{q}_{1} \dot{\xi}_{0}\right) \tan q_{3}-\frac{\dot{q}_{1}}{\cos ^{2} q_{3}} \xi_{3}=0$.

Identity (14) becomes

$$
\begin{aligned}
& L \dot{\xi}_{0}+m \dot{q}_{1}\left(\dot{\xi}_{1}-\dot{q}_{1} \dot{\xi}_{0}\right)+m \dot{q}_{2}\left(\dot{\xi}_{2}-\dot{q}_{2} \dot{\xi}_{0}\right)+J \dot{q}_{3}\left(\dot{\xi}_{3}-\dot{q}_{3} \dot{\xi}_{0}\right) \\
& \quad-k q_{3} \xi_{3}-m \dot{q}_{1} \dot{q}_{3}\left(\xi_{1}-\dot{q}_{1} \xi_{0}\right) \tan q_{3}+m \dot{q}_{1} \dot{q}_{3}\left(\xi_{2}-\dot{q}_{2} \xi_{0}\right) \\
& \quad+\dot{G}_{N}=0 .
\end{aligned}
$$

Equation (15) provide

$\xi_{2}-\dot{q}_{2} \xi_{0}-\left(\xi_{1}-\dot{q}_{1} \xi_{0}\right) \tan q_{3}=0$.

These six equations have the solution

$\xi_{0}=-1, \quad \xi_{1}=\xi_{2}=\xi_{3}=0, \quad G_{N}=0$,

and the conserved quantity (43) gives

$I_{N}=\frac{1}{2} m\left(\dot{q}_{1}^{2}+\dot{q}_{2}^{2}\right)+\frac{1}{2} J \dot{q}_{3}^{2}+\frac{1}{2} k q_{3}^{2}=$ const.

which is the integral of energy of the system.

\subsection{Birkhoffian system}

For the Birkhoffian system, the Lie symmetry can lead to the Noether conserved quantity and the new conserved quantity. We have

Proposition 9.7 For the Birkhoffian system (17), if the generators $\xi_{0}, \xi_{\mu}$ of the Lie symmetry and the gauge function $G_{N}=G_{N}(t, a)$ satisfy the Noether identity (20), then the Lie symmetry will lead to the Noether conserved quantity (45).

Proposition 9.8 For the Birkhoffian system (17), if the generators $\xi_{0}, \xi_{\mu}$ of the Lie symmetry satisfy Eq. (42), and the generators and the gauge function $G_{F}=G_{F}(t, a)$ satisfy Eq. (69), then the Lie symmetry will lead to the new conserved quantity (71). 
Example 9.4 The Birkhoffian and the Birkhoff's functions of a Birkhoffian system of four order are respectively

$B=\left(a^{2} \sin t+a^{4} \cos t\right)^{2}$,

$R_{1}=0, \quad R_{2}=a^{1}-a^{4}-\left(a^{2}+a^{3}\right) t$

$$
+\left(a^{2} \sin t+a^{4} \cos t\right) \cos t,
$$

$R_{3}=a^{1}-a^{4}-\left(a^{2}+a^{3}\right) t$,

$R_{4}=-\left(a^{2} \sin t+a^{4} \cos t\right) \sin t$.

Equation (31) give

$\dot{\xi}_{1}-a^{3} \dot{\xi}_{0}=\xi_{3}, \quad \dot{\xi}_{2}-a^{4} \dot{\xi}_{0}=\xi_{4}$,

$\dot{\xi}_{3}+a^{4} \dot{\xi}_{0}=-\xi_{4}, \quad \dot{\xi}_{4}+a^{2} \dot{\xi}_{0}=-\xi_{2}$.

which have the solution

$\xi_{0}=\xi_{2}=\xi_{4}=0, \quad \xi_{1}=\left(a^{2}+a^{3}\right) t+1, \quad \xi_{3}=a^{2}+a^{3}$,

and then we have

$X^{(0)}(B)=X^{(0)}\left(R_{1}\right)=X^{(0)}\left(R_{4}\right)=0$,

$X^{(0)}\left(R_{2}\right)=X^{(0)}\left(R_{3}\right)=1$,

$G_{F}=0$.

The conserved quantity (71) gives

$I_{F}=a^{2}+a^{3}=$ const

\section{Conserved quantities deduced by form invariance}

In Sect. 7, we have presented the new conserved quantity deduced by the form invariance for the constrained mechanical systems. In fact, the Noether conserved quantity and the Hojman type conserved quantity can also be deduced by using the form invariance for the systems.

\subsection{Lagrangian system}

Proposition 10.1 For the Lagrangian system (1), if the generators $\xi_{0}, \xi_{s}$ of the form invariance and the gauge function $G_{N}=G_{N}(t, \boldsymbol{q}, \dot{\boldsymbol{q}})$ satisfy the Noether identity (6), then the form invariance will lead to the Noether conserved quantity (43).

Proposition 10.2 For the Lagrangian system (1), if the generators $\xi_{s}$ of the form invariance satisfy Eq. (47), and there exists a function $\mu=\mu(t, \boldsymbol{q}, \dot{\boldsymbol{q}})$ satisfying $E q$. (49), then the form invariance will lead to the Hojman type conserved quantity (50).

Example 10.1 The Lagrangian of a system is

$L=\frac{1}{2}\left(\dot{q}_{1}^{2}+\dot{q}_{2}^{2}\right)+\frac{1}{b} \dot{q}_{1} \arctan b t+\frac{1}{2 b} \dot{q}_{2} \ln \left(1+b^{2} t^{2}\right)$, where $b$ is a constant.
Taking the generators as

$\xi_{0}=\xi_{2}=0$

$\xi_{1}=\frac{1}{2}\left\{q_{1}+\int \frac{1}{b} \arctan b t \mathrm{~d} t-t\left[\dot{q}_{1}+\frac{1}{b} \arctan b t\right]\right\}^{2}$,

and then

$X^{(1)}(L)=0$,

therefore the generators are form invariance ones. In this case, Eq. (47) are verified. Equation (49) gives

$\frac{\overline{\mathrm{d}}}{\mathrm{d} t} \ln \mu=0$,

and we have

$\mu=1$.

The Hojman type conserved quantity (50) gives

$$
\begin{aligned}
I_{H} & =q_{1}+\int \frac{1}{b} \arctan b t \mathrm{~d} t-t\left[\dot{q}_{1}+\frac{1}{b} \arctan b t\right] \\
& =\text { const. }
\end{aligned}
$$

10.2 General holonomic system

Proposition 10.3 For the general holonomic system (8), if the generators $\xi_{0}$, $\xi_{s}$ of the form invariance and the gauge function $G_{N}=G_{N}(t, \boldsymbol{q}, \dot{\boldsymbol{q}})$ satisfy the Noether identity (9), then the form invariance will lead to the Noether conserved quantity (43).

Proposition 10.4 For the general holonomic system (8), if the generators $\xi_{s}$ of the form invariance satisfy Eq. (51) and there exists a function $\mu=\mu(t, \boldsymbol{q}, \dot{\boldsymbol{q}})$ satisfying Eq. (52), then the form invariance will lead to the Hojman type conserved quantity (50).

Example 10.2 The Lagrangian and the generalized forces of a system with two-degree-of-freedom are respectively

$L=\frac{1}{2}\left(\dot{q}_{1}^{2}+\dot{q}_{2}^{2}\right)-f\left(t, q_{2}\right)$,

$Q_{1}=-\dot{q}_{2}, \quad Q_{2}=Q_{2}\left(t, q_{2}, \dot{q}_{2}\right)$.

Taking the generators as

$\xi_{0}=\xi_{2}=0, \quad \xi_{1}=1$

and we have

$X^{(1)}(L)=X^{(1)}\left(Q_{1}\right)=X^{(1)}\left(Q_{2}\right)=0$.

Identity (9) gives

$G_{N}=q_{2}$,

and the conserved quantity (43) gives

$I_{N}=\dot{q}_{1}+q_{2}=$ const. 
which is a Noether conserved quantity deduced by the form invariance.

\subsection{Nonholonomic system}

Proposition 10.5 For the nonholonomic system (10), (11), if the generators $\xi_{0}, \xi_{s}$ of the form invariance and the gauge function $G_{N}=G_{N}(t, \boldsymbol{q}, \dot{\boldsymbol{q}})$ satisfy the Noether identity (14) and Eq. (15), then the form invariance will lead to the Noether conserved quantity (43).

Proposition 10.6 For the nonholonomic system (10), (11), if the generators $\xi_{s}$ of the form invariance satisfy Eq. (53) and (54), and there exists a function $\mu=\mu(t, \boldsymbol{q}, \dot{\boldsymbol{q}})$ satisfying $E q$. (55), then the form invariance will lead to the Hojman type conserved quantity (50) [65].

Example 10.3 Novoselov problem is

$L=\frac{1}{2}\left(\dot{q}_{1}^{2}+\dot{q}_{2}^{2}\right), \quad f=\dot{q}_{1}+b t \dot{q}_{2}-b q_{2}+t=0$.

Taking the generators as

$\xi_{0}=0, \quad \xi_{1}=\left(\dot{q}_{2}-b t \dot{q}_{1}+b q_{1}\right)^{2}, \quad \xi_{2}=0$,

we can verify that the generators are form invariant and Lie symmetric. Then Eq. (55) gives

$\mu=1$,

and the conserved quantity (50) gives

$I_{H}=2 b\left(\dot{q}_{2}-b t \dot{q}_{1}+b q_{1}\right)=$ const.

\subsection{Birkhoffian system}

Proposition 10.7 For the Birkhoffian system (17), if the generators $\xi_{0}, \xi_{\mu}$ of the form invariance and the gauge function $G_{N}=G_{N}(t, a)$ satisfy the Noether identity (20), then the form invariance will lead to the Noether conserved quantity (45).

Proposition 10.8 For the Birkhoffian system (17), if the generators $\xi_{\mu}$ of the form invariance satisfy Eq. (57), and there exists a function $\mu=\mu(t, \boldsymbol{a})$ satisfying $E q$. (59), then the form invariance will lead to the Hojman type conserved quantity (60).

Example 10.4 The Birkhoffian and the Birkhoff's functions of a Birkhoffian system are respectively

$B=\frac{1}{2} \frac{1}{m+f(t)}\left\{k_{2}\left(a^{2}\right)^{2}-k_{1}\left(a^{1}\right)^{2}\right\}$,

$R_{1}=a^{2}, \quad R_{2}=0$,

where $m, k_{1}$ and $k_{2}$ are constants.
Taking the generators as

$\xi_{0}=0, \quad \xi_{1}=k_{2} a^{2}, \quad \xi_{2}=k_{1} a^{1}$,

we can verify that they are form invariance generators. Identity (20) gives

$G_{N}=-\frac{1}{2}\left\{k_{1}\left(a^{1}\right)^{2}+k_{2}\left(a^{2}\right)^{2}\right\}$,

and the conserved quantity (45) gives

$I_{N}=\frac{1}{2}\left\{k_{2}\left(a^{2}\right)^{2}-k_{1}\left(a^{1}\right)^{2}\right\}=$ const.

The methods for finding the conserved quantities in the review are called the symmetry methods. The symmetry methods are important modern integration methods in dynamics. By using the methods, one can find not only the conserved quantities deduced by Newtonian mechanics and Lagrangian mechanics, but also the conserved quantities which can not be deduced by them. Of course, when using the methods the mathematical difficulties can crop up. The complexity in the Noether symmetry method is to seek the generators and the gauge function by the Noether identity. The complexity in the Lie symmetry method is to find the generators in the determining equations and the function $\mu$, and the difficulty in the form invariance method is to find the generators and the gauge function in the structure equation. The Noether symmetry method is more convenient than the two others, because a Noether symmetry corresponds, in general, to a Noether conserved quantity.

We have presented three kinds of symmetries, i.e. the Noether symmetry, the Lie symmetry and the form invariance, three kinds of conserved quantities, i.e. the Noether conserved quantity, the Hojman type conserved quantity and the new conserved quantity, for the Lagrangian system, the general holonomic system, the nonholonomic system and the Birkhoffian system. Every symmetry can lead to three kinds of conserved quantities. One can study the conserved quantity of other constrained mechanical systems, say, the Hamiltonian system, the holonomic system with redundant coordinates, the variable mass system, the dynamical system in the event space, the generalized Hamiltonian system, the nonholonomic system with the nonholonomic constraints of non-Chetaev's type, the vakonomic system, etc.

There is a symmetry involving equivalent Lagrangians of the Lagrangian system and a conserved quantity can be found by using the symmetry [66]. This idea can be generalized to other constrained mechanical systems. There is a symmetry under which a non-Noether conserved quantity can be found $[57,67,68]$. The mathematical tools used in the review are simpler analytical ones. There are differential geometry methods in the study of the symmetry, e.g. see references $[15,19,69-81]$. 


\section{Open problems}

Below, we summarize some problems for future research in the area of the symmetry and the conserved quantity of constrained mechanical systems:

1. Find new symmetry methods;

2. Solve the general differential equations by using the symmetry methods;

3. Study the stability of motion by using the symmetry methods;

4. Study the symmetry and the conserved quantity of the vakonomic system.

Acknowledgments This work was supported by the National Natural Science Foundation of China (Grant Nos. 10932002, 11272050).

\section{References}

1. Noether E (1918) Invariante variationsprobleme. Kgl Ges Wiss Nachr Göttingen. Math Phys KI:235-257

2. Dass T (1966) Conservation laws and symmetries II. Phys Rev 150:1251-1255

3. Parmieri C, Vitale B (1970) On the inversion of Noether's theorem in the Lagrangian formalism. Nuovo Cimento 66A:299-309

4. Candotti E, Parmieri C, Vitale B (1972) On the inversion of Noether's theorem in classical dynamical systems. Am J Phys 40:424-429

5. Djukić Dj D, Vujanović B (1975) Noether's theory in classical nonconservative mechanics. Acta Mech 23:17-27

6. Desloge EA, Karch RI (1977) Noether's theorem in classical mechanics. Am J Phys 45:336-339

7. Vujanović B (1978) Conservation laws of dynamical systems via d'Alembert's principle. Int J Non-Linear Mech 13:185-197

8. Vujanović B (1986) A study of conservation laws of dynamical systems by means of differential variational principles of Jourdain and Gauss. Acta Mech 65:63-80

9. Sarlet W, Cantrijn F (1981) Higher-order Noether symmetries and constantsof the motion. J Phys A Math Gen 14:479-492

10. Li Z-P (1981) Transformation property of constrained system (in Chinese). Acta Phys Sin 30:1659-1671

11. Kalotas TM, Wybourne BG (1982) Dynamical Noether symmetries. J Phys A Math Gen 15:2077-2083

12. Bahar LY, Kwatny HG (1987) Extension of Noether's theorem to constrained nonconservative dynamical systems. Int J Non-Linear Mech 22:125-138

13. Liu D (1991) Noether's theorem and its inverse of nonholonomic nonconservative dynamical systems. Sci China 34:419-429

14. Mei F-X (1993) The Noether's theory of Birkhoff systems. Sci China 36:1456-1467

15. Liu C, Zhao Y-H, Chen X-W (2010) Geometric representation of Noether symmetry for dynamical systems. Acta Phys Sin 59:11-14 (in Chinese)

16. Freire IL, da Silva PL, Torrisi M (2013) Lie and Noether symmetries for a class of fourth-order Emden-Fowler equations. J Phys A Math Theory 46:245206

17. Zhang Y, Zhou Y (2013) Symmetries and conserved quantities for fractional action-like Pfaffian variational problems. Nonlinear Dyn 73:783-793

18. Wang P, Xue Y, Liu Y-L (2013) Noether symmetry and conserved quantities of the analytical dynamics of a Cosserat thin elastic rod. Chin Phys B 22:104503
19. Arnold VI (1978) Mathematical methods of classical mechanics. Springer, New York

20. José JV, Saletan EJ (1998) Classical dynamics: a contemporary approach. Cambridge University Press, Cambridge

21. Whittaker ET (1937) A treatise on the analytical dynamics of particles and rigid bodies, 4th edn. Cambridge University Press, Cambridge

22. Hamel G (1949) Theoretische mechanik. Springer, Berlin

23. Appell P (1953) Traité de mécanique rationnelle II. GauthierVillars, Paris

24. Lur'e AI (1961) Analytical mechanics (in Russian). Gostecnizdat, Moscow

25. Novoselov VS (1966) Variational methods in mechanics (in Russian). Leningrad University Press, Leningrad

26. Neimark Ju I, Fufaev NA (1967) Dynamics of nonholonomic systems (in Russian). Nauka, Moscow

27. Dobronravov VV (1970) Elements of mechanics of nonholonomic systems (in Russian). Vischaya Shkola, Moscow

28. Rumyantsev VV (1983) On the problems of analytical mechanics of nonholonomic systems. In: Proceedings of IUTAM-ISIMM symposium on modern developments in analytical mechanics, pp 697-716

29. Polyakhov NN, Zegzhda SA, Yushkov MP (1985) Theoretical mechanics (in Russian). Leningrad University Press, Leningrad

30. Mei F-X (1985) Foundations of mechanics of nonholonomic systems (in Chinese). Beijing Institute of Technology Press, Beijing

31. Papastavridis JG (2002) Analytical mechanics. Oxford University Press, Oxford

32. Mei F-X (2000) Nonholonomic mechanics. Appl Mech Rev (ASME) 53:283-305

33. Papastavridis JG (1998) A panoramic overview of the principles and equations of motion of advanced engineering dynamics. Appl Mech Rev (ASME) 51:239-265

34. Santilli RM (1983) Foundations of theoretical mechanics, vol II. Springer, New York

35. Mei F-X (2001) On the Birkhoffian mechanics. Int J Non-Linear Mech 36:817-834

36. Olver PJ (1986) Applications of Lie groups to differential equations. Springer, New York

37. Bluman GW, Kumei S (1989) Symmetries and differential equations. Springer, New York

38. Ibragimov NH (1994) CRC handbook of Lie group analysis of differential equations. CRC Press, Boca Raton

39. Bluman GW, Anco SC (2002) Symmetry and integration methods for differential equations. Springer, New York

40. Lutzky M (1979) Dynamical symmetries and conserved quantities. J Phys A Math Gen 12:973-981

41. Prince GE, Leach PGL (1980) The Lie theory of extended groups in Hamiltonian mechanics. Hadronic J 3:941-961

42. Prince GE, Eliezer CJ (1981) On the Lie symmetries of the classical Kepler problem. J Phys A Math Gen 14:587-596

43. Hojman SA (1992) A new conservation law constructed without using either Lagrangians or Hamiltonians. J Phys A Math Gen 25:L291-L295

44. Zhao Y-Y (1994) Conservative quantities and Lie symmetries of nonconservative dynamical systems (in Chinese). Acta Mech Sin 26:380-384

45. Mei F-X (2000) Lie symmetries and conserved quantities of constrained mechanical systems. Acta Mech 141(3-4):135-148

46. Zheng S-W, Wang J-B, Chen X-W, Li Y-M, Xie J-F (2012) Lie symmetry and their conserved quantities of Tznoff equations for the variable mass nonholonomic systems. Acta Phys Sin 61:111101 (in Chinese)

47. Wang X-Z, Fu H, Fu J-L (2012) Lie symmetries and conserved quantities of discrete nonholonomic Hamiltonian systems. Chin Phys B 21:040201 
48. Han Y-L, Wang X-X, Zhang M-L, Jia L-Q (2013) Lie symmetry and approximate Hojman conserved quantity of Appell equations for a weakly nonholonomic system. Nonlinear Dyn 71:401-408

49. Katzin GH, Levine J (1985) Characteristic functional structure of infinitesimal symmetry mapping of classical dynamical systems I: velocity-dependent mappings of second-order differential equations. J Math Phys 26:3080-3098

50. Mei F-X, Zheng G-H (2002) On the Noether symmetry and the Lie symmetry of mechanical systems. Acta Mech Sin 18:414-419

51. Mei F-X (2000) Form invariance of Lagrange system. J Beijing Inst Technol 9:120-124

52. Wang S-Y, Mei F-X (2002) Form invariance and Lie symmetry of equations of nonholonomic systems. Chin Phys 11:5-8

53. Chen X-W, Luo S-K, Mei F-X (2002) A form invariance of constrained Birkhoffian system. Appl Math Mech 23:53-57

54. Xu X-J, Mei F-X, Qin M-C (2004) Non-Noether conserved quantity constructed by using form invariance for Birkhoffian system. Chin Phys 13:1999-2002

55. Wu H-B, Mei F-X (2004) Form invariance and Lie symmetry of the generalized Hamiltonian system. Acta Mech Solida Sin 17:370 373

56. Fu J-L, Chen L-Q (2004) On the Noether symmetries and form invariances of mechanical-electrical systems. Phys Lett A 331:138-152

57. Mei F-X, Xu X-J (2005) Form invariance and Lutzky conserved quantities for Lagrange systems. Chin Phys 14:449-451

58. Jiang W-A, Luo S-K (2011) Mei symmetry leading to Mei conserved quantity of generalized Hamiltonian system. Acta Phys Sin 60:060201 (in Chinese)

59. Cai J-L (2012) Conformal invariance of Mei symmetry for the nonholonomic systems of non-Chetaev's type. Nonlinear Dyn 69:487493

60. Zhao G-L, Chen L-Q, Fu J-L, Hong F-Y (2013) Mei symmetry and conservation laws of discrete nonholonomic dynamical systems with regular and irregular lattices. Chin Phys B 22:030201

61. Xia L-L, Chen L-Q (2013) Conformal invariance of Mei symmetry for discrete Lagrangian systems. Acta Mech 224:2037-2043

62. Lou Z-M (2003) Conserved quantity of isotopic harmonic oscillator determining by the Noether theory (in Chinese). Mech Eng 25:7273

63. Lucas WF (1983) Differential equation models. Springer, New York

64. Luo S-K, Mei F-X (2004) A non-Noether conserved quantity, i.e. Hojman conserved quantity, for nonholonomic mechanical systems (in Chinese). Acta Phys Sin 53:666-670
65. Luo S-K, Guo Y-X, Mei F-X (2004) Noether symmetry and Hojman conserved quantity for nonholonomic mechanical systems (in Chinese). Acta Phys Sin 53:1271-1275

66. Hojman SA, Harleston H (1981) Equivalent Lagrangians: multidimensional case. J Math Phys 22:1414-1419

67. Lutzky M (1979) Non-invariance symmetries and constants of the motion. Phys Lett A 72:86-88

68. Fu J-L, Chen L-Q (2003) Non-Noether symmetries and conserved quantities of nonconservative dynamical systems. Phys Lett A 317:255-259

69. Godbillon C (1969) Géométrie differentielle et mécanique analytique. Hermann, Paris

70. Souriau JM (1969) Structure des systèmes dynamiques. Dunod, Paris

71. Tulczyjew WM (1976) Lagrangian submanifolds and Hamiltonian dynamics. CR Acad Paris 283:15-18

72. Abraham R, Marsden JE (1978) Foundations of mechanics. Benjamin/Cummings Publ Co, London

73. Benenti S (1983) Symplectic relation in analytical mechanics. In: Proceedings of IUTAM-ISIMM symposium on modern developments in analytical mechanics, pp 39-91

74. Weber RM (1986) Hamiltonian systems with constraints and their meaning in mechanics. Arch Rational Mech Anal 91:309-335

75. Cantrijn F, Carinena J, Crampin M, Ibort L (1986) Reduction of degenerate Lagrange systems. J Geom Phys 3:353-400

76. De León M, Rodrigues PR (1989) Methods of differential geometry in analytical mechanics. North-Holland, Amsterdam

77. Sarlet W, Prince GE, Crampin M (1990) Adjoint symmetries for time-dependent second-order equations. J Phys A Math Gen 23:935-947

78. Vershik AM, Gershkovich VYa (1994) Nonholonomic dynamics, geometry of distributions and variational problems. Dyn Syst 7:181

79. Marsden JE, Ratiu TS (1994) Introduction to mechanics and symmetry. Springer, New York

80. Bloch AM, Krishnaprasad PS, Marsden JE, Murray RM (1996) Nonholonomic mechanical systems with symmetry. Arch Rational Mech Anal 136:21-99

81. Guo Y-X, Shang M, Luo S-K, Mei F-X (2001) Poincaré-Cartan integral variants and invariants of nonholonomic constrained systems. Int J Theor Phys 40:1197-1205 